\title{
Information and Communication Theoretical Understanding and Treatment of Spinal Cord Injuries: State-of-the-art and Research Challenges
}

\author{
Ozgur B. Akan, Fellow, IEEE, Hamideh Ramezani, Student Member, IEEE, Meltem Civas, Student \\ Member, IEEE, Oktay Cetinkaya, Member, IEEE, Bilgesu A. Bilgin, Member, IEEE and Naveed A. \\ Abbasi, Student Member, IEEE
}

\begin{abstract}
Among the various key networks in the human body, the nervous system occupies central importance. The debilitating effects of spinal cord injuries (SCI) impact a significant number of people throughout the world, and to date, there is no satisfactory method to treat them. In this paper, we review the major treatment techniques for SCI that include promising solutions based on information and communication technology (ICT) and identify the key characteristics of such systems. We then introduce two novel ICT-based treatment approaches for SCI. The first proposal is based on neural interface systems (NIS) with enhanced feedback, where the external machines are interfaced with the brain and the spinal cord such that the brain signals are directly routed to the limbs for movement. The second proposal relates to the design of self-organizing artificial neurons (ANs) that can be used to replace the injured or dead biological neurons. Apart from SCI treatment, the proposed methods may also be utilized as enabling technologies for neural interface applications by acting as bio-cyber interfaces between the nervous system and machines. Furthermore, under the framework of Internet of BioNano Things (IoBNT), experience gained from SCI treatment techniques can be transferred to nano communication research.
\end{abstract}

Index Terms-Spinal cord injuries, spinal treatments, neural interface systems, artificial neurons.

\section{INTRODUCTION}

The nervous system is one of the most important networks of the body that forms the basis of human intellect and stores our experiences as memories. It is composed of networks of neurons connected through synapses to receive, transmit, and process information in the body. The manipulation of information by the nervous system is still not entirely understood. Thus, an information and communication technology (ICT) framework is required to evaluate the relationships of information exchange between various entities of the nervous system, and then to address the associated problems [1].

O. B. Akan is with the Internet of Everything (IoE) Group, Department of Engineering, University of Cambridge, UK and the Next-generation and Wireless Communications Laboratory (NWCL), College of Engineering, Koc University, Istanbul, Turkey. (e-mail: oba21@cam.ac.uk.)

H. Ramezani and B. A. Bilgin are with the Internet of Everything (IoE) Group, Electrical Engineering Division, Department of Engineering, University of Cambridge. (e-mails: $\{$ hr404, bab46 $\} @$ cam.ac.uk.)

M. Civas, O. Cetinkaya, and N. A. Abbasi are with the Next-generation and Wireless Communications Laboratory (NWCL), Department of Electrical and Electronics Engineering, Koc University, Istanbul, Turkey (e-mails: \{mcivas16, okcetinkaya13, nabbasi13\}@ku.edu.tr).

This work was supported in part by ERC project MINERVA (ERC-2013CoG \#616922), AXA Chair for Internet of Everything and Huawei Graduate Research Scholarship.
TABLE I: Types of SCI and Corresponding Percentages.

\begin{tabular}{|l|c|}
\hline SCI Types & Percentage \\
\hline \hline Incomplete Tetraplegia & $45 \%$ \\
\hline Incomplete Paraplegia & $21.3 \%$ \\
\hline Complete Paraplegia & $20 \%$ \\
\hline Complete Tetraplegia & $13.3 \%$ \\
\hline Recovery & $0.4 \%$ \\
\hline
\end{tabular}

Communication between the brain and limbs is maintained by the spinal cord, which is comprised of ascending and descending spinal pathways [2]. Each spinal pathway constitutes of neurons having long axons, projecting between the brain and spinal cord. In patients with spinal cord injuries (SCI), advanced amyotrophic lateral sclerosis (ALS), or brainstem strokes, the brain is functional; however, its signals are not transmitted to the muscles due to interruption in the transmission pathways.

The social and economic burden of SCI is globally extensive. According to the findings [3], 0.93 million new cases of SCI occurred in 2016, while prevalent cases were 27.04 million. SCI results in partial loss of motor and/or sensory functions in the incomplete injury. In the case of complete SCI, the patient suffers from the total loss of motor and sensory abilities. Statistics for the United States provided in Table I reveal that recovery after injury is very unlikely, and in most cases SCI causes neurological deficits in all limbs and torso, i.e., tetraplegia [4]. In the less severe form of SCI, paraplegia, the arms are not affected.

Two main approaches exist for re-establishing the brainbody connection of the patients suffering from SCI, which we group as, biological methods and ICT-based treatment techniques. The latter is the main interest of this paper since it has advantageous aspects in terms of functional outcome, human trials, and safety. The ICT-based treatment techniques of SCI can be achieved by (i) the use of neural interface systems (NIS), and (ii) deployment of artificial neurons (ANs). NIS were first developed for animals [5]-[10] and then utilized for human patients to extract control signals from nervous system and operate assistive devices, including computer cursors [11] [15] and robotic prostheses [16]-[19]. More importantly, they were also used to apply electrical stimulations to the nervous system or muscles for restoring motor capabilities [20]-[25], which can induce partial neurological recovery [26], [27]. The 
deployment of ANs, on the other hand, targets to replace injured or dead biological neurons. This is a relatively recent direction, and there is still no major work that can qualify itself as a fully-functional AN.

In this paper, we first discuss the mechanisms of SCI to give some insight on these injuries. We then present the existing treatment techniques including biological, NIS, and AN-based approaches. Specifically, for the NIS and the AN, where the ICT techniques may benefit from the existing designs, we also identify the key characteristics of a successful treatment technique. Based on the preceding findings and discussions, we then present two potential solutions that may be able to treat SCI with their respective methodologies. The first approach is based on NIS, where we identify key areas for improvement in the existing setups. We point out important areas, such as feedback, that are yet unexplored. The second proposal is for the development of ANs based on nanomachines that act as a bridge to deliver neural signals across the injury site. The proposed AN will incorporate plasticity along with selforganization, and will thus be able to adapt in the injury area to make new nervous connections. Additionally, the machines are proposed to include energy harvesting $(\mathrm{EH})$ so that they are truly independent in nature. Apart from SCI treatment, the proposed schemes can be used as bio-cyber interfaces in a variety of applications and also motivate for Internet of Bio-Nano Things (IoBNT) and intra-body nanonetworks applications.

The rest of this paper is organized as follows. In Section II, we present an overview of nervous communication, the mechanisms for SCI, and some key treatment approaches. The existing ICT-based treatment techniques for SCI are detailed in Section III. Section IV introduces our proposed methods, whereas Section V provides some future directions in SCI treatment as well as other potential applications for the SCI treatment techniques. Finally, Section VI concludes the paper.

\section{SpinAl CoRd InJURy TREATMEnt TeChNiques}

In this section, we present an overview of nervous system communication, how it is disrupted by SCI during various phases of the injuries, the existing biological treatment techniques for SCI, and the key differences between the biological and ICT-based treatment techniques.

\section{A. Neuro-spike Communication}

Communication in the ultra large-scale nervous network, known as neuro-spike communication, takes place through electrical or chemical synapses with the latter occurring more frequently [28]. Information encoded in electrical impulses is transmitted to the post-synaptic neuron using three steps. (i) Axonal transmission: the electrical impulses pass through the axon of the pre-synaptic neuron until they reach the presynaptic terminals. (ii) Synaptic propagation: arrival of an electrical impulse to the pre-synaptic terminal initiates the release of neurotransmitter into the synaptic cleft, i.e., the gap between input and output neuron. The neurotransmitters then diffuse though the synaptic cleft to reach the post-synaptic neuron and bind to the receptors located on its membrane. This

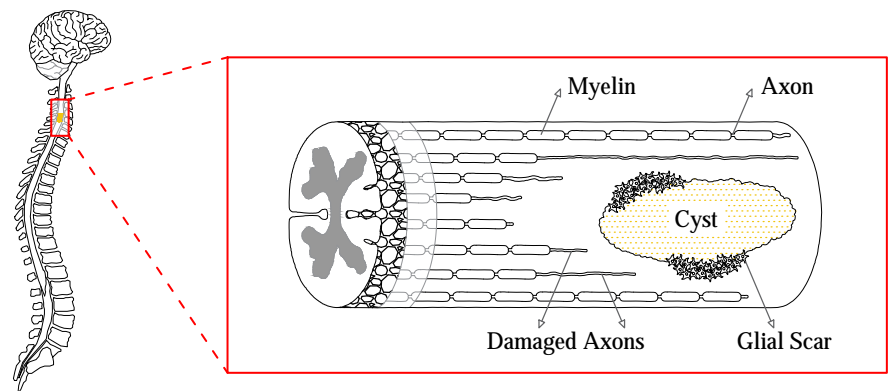

Fig. 1: SCI and the formation of glial scars. The demyelination, i.e., the loss of myelin sheath, occurring due to neuroinflammation significantly affects the axonal propagation, which might get disrupted in severe cases.

changes the post-synaptic membrane potential. (iii) Spike generation: Depending on the released neurotransmitters, changes in post-synaptic membrane potential can be both excitatory, i.e., a temporary increase in the postsynaptic membrane potential caused by the flow of positively charged ions into the postsynaptic cell, or inhibitory, which makes a postsynaptic neuron less likely to generate an action potential. Postsynaptic potentials are subject to spatial and temporal summation. Spatial summation occurs when a neuron is receiving input from multiple synapses that are near each other. Temporal summation occurs when a neuron receives inputs that are close together in time, i.e., the next change in membrane potential happens before the previous change is vanished. If the total change in the post-synaptic membrane potential due to all excitatory and inhibitory synapses is greater than the spiking threshold, the post-synaptic neuron fires an electrical impulse.

Several studies exist in the literature on modeling and analyzing the performance of neuro-spike communication [28][36] and the nervous nanonetwork [37], [38] considering the functionality of healthy neurons. Any disruption in the functionality of neurons, which can result from diseases or injuries, adversely affects the performance of this nanoscale communication network.

\section{B. Spinal Cord Circuitry}

The nervous system comprises two main systems: the peripheral and central nervous networks. A part of the peripheral nervous network (PNN) called somatic nervous network (SNN) is related to the control of voluntary body movements. Voluntary body movements are controlled by conveying sensory inputs to central nervous network (CNN) via sensory nerves and motor outputs from $\mathrm{CNN}$ to the skeletal muscles by motor nerves. Motor centers in the brain generate motor outputs which are integrated by spinal cord with the sensory inputs. The response generated by the spinal cord is transmitted to the muscles [39]. Spinal cord circuitry consists of several complex networks, which can be divided as motor network and sensory network from the communication theory perspective.

Motor network performs the downlink transmission of the motor commands from the brain to the muscles. As depicted in Fig. 2(a) cortical neurons transmit motor inputs to the spinal cord interneurons, which integrate motor inputs conveyed from 


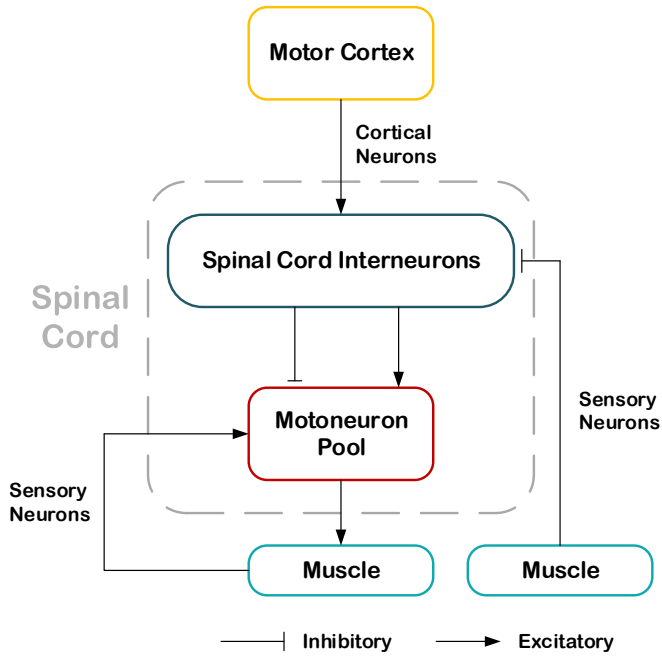

(a) Diagram showing a spinal cord motor network.

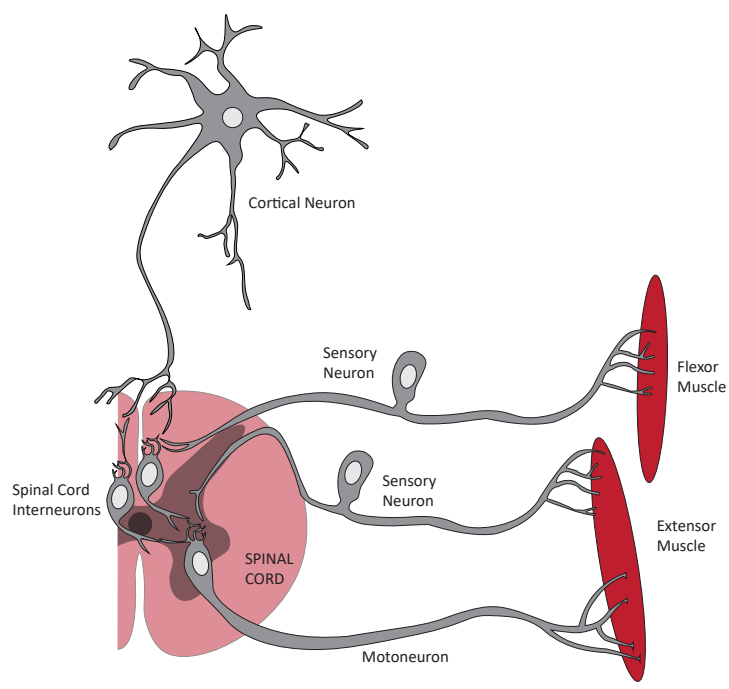

(b) A spinal cord interneuronal network.

Fig. 2: Diagram illustrating spinal cord sensory-motor networks [40].

the brain and the sensory inputs transmitted from the sensory organs in the muscles. In primates, cortical neurons directly project to motoneurons [41]. A motoneuron pool in the spinal cord is the final destination for the sensory-motor inputs controlling a specific muscle. Thus, motoneurons are important for both fine and gross motor control. Moreover, they connect to sensory neurons via the spinal interneurons in reflex activity.

Sensory network controls the uplink transmission of sensory information from the skeletal muscles to the brain by using sensory pathways comprising sensory nerves. Sensory modalities from the body are detected by afferent fibers whose cell bodies reside in dorsal root ganglion. These primary afferents mostly terminate in the spinal cord, where sensory information is first processed and synapse with interneurons or projection neurons forming tracts. Projections that course through ascending pathways convey messages to higher targets in the central nervous network including brainstem and somatosensory cortex.

In the case of damage to the cortical neurons with long axons projecting to the spinal cord as a result of SCI, the motor inputs for the voluntary movements may not be delivered reliably to the spinal cord, where the spinal cord interneurons integrate the motor inputs from the brain and the sensory outputs from the muscles. To activate the spinal cord interneuron circuit, the motor commands read from the motor cortex area related to fine and grass motor movements can be delivered artificially to the spinal cord interneuron circuits. Considering SCI resulting in a damage to the sensory network, the sensory signal can be read from the dorsal root ganglion, which contains the nerve bundles of first order sensory neurons. Then, the sensory signal obtained can be delivered to the related parts of the somatosensory cortex. To differentiate the sensory signals from each other signal processing techniques can be employed.

\section{Injury Mechanisms}

Neurons inflicted by spinal trauma experience the effects of an injury in three consecutive phases, namely, primary, secondary, and chronic injuries described below.

- Primary injury: This refers to the condition within minutes after the injury happens. The injury immediately disrupts axonal pathways and damages cell membranes depending on the physical trauma [42]. The ischaemia, i.e., toxic chemical release from damaged membranes, initiates the secondary injury [42]. Many active neural connections, and thus neurospike communications, are affected by this extracellular toxicity.

- Secondary injury: Following hours to weeks after the injury, SCI develops a condition called secondary injury [43]. In this phase, deteriorated toxic environment in the spinal cord causes death of neurons as well as glial cells. [44]. Glial cells produce myelin sheath, which acts as an insulator and improves the rate of information transmission along the axon. The loss of these special cells significantly affects axonal propagation due to loss of myelin sheath, namely demyelination [45]. Moreover, axonal regeneration is blocked by glial scar formation, which is a tissue barrier formed in the injury site, as depicted in Fig. 11. In severe cases, axonal transmission is blocked due to demyelination and disruption of the brain-spinal cord communication [45].

- Chronic injury: This phase covers the days to years following the injury [43]. In this phase, injured axons experience a special kind of degeneration, called Wallerian degeneration, that disconnects the distal parts of the axons from their cell bodies while the glial scar continues to grow [46]. Wallerian degeneration consists of several phases, in which axonal death occurs and axon debris is cleared by the glial cells [47]. However, the proximal parts of the motor fibers are preserved and still functioning. The time course of the degeneration of cell bodies is very slow compared to that 
of axonal degeneration. To illustrate, even years after the injury, cell bodies can be preserved [48]. This makes the recording motor signals from the spinal cord descending tracts possible [48]. Any damage to the long axons of descending spinal cord tract conveying information of motor actions will damage to the spinal cord motor network. However, the circuitry below the injury will remain intact.

The overall result of SCI mechanisms is the disruption of axonal pathways located within the spinal cord, which results in either the loss of sensory information transmission to the brain or motor control signals to the muscles or both at the same time [42]. Hence, re-establishing the brain-spinal cord communication is necessary to maintain the sensory and motor functions of the nervous nanonetwork. As discussed earlier, current treatment approaches fall in two main categories, namely the biological and the ICT-based treatment techniques. In the following, we discuss the major techniques that belong to these categories and the key differences between them.

\section{Biological Treatment Techniques}

Following the initial disruption of SCI, damage continues to spread in the spinal cord in the secondary and the chronic phases. Despite to ongoing research and clinical trials on preventing the effects of pathological results of SCI, a gold standard treatment of SCI is not known [49]. Preventive and treatment strategies can be classified as biological and ICTbased approaches. Biological treatment approaches aims to protect surviving neurons from the toxic post-injury environment, and to recover synaptic connections and healthy functionality by promoting regeneration. On the contrary, ICT-based treatment techniques directly address the neural communication problem by either bypassing the injury site via external communication interfaces or by replacing the injured neurons by artificially designed neurons.

Next, we outline the existing fundamental biological treatment approaches and the challenges faced regarding their implementation. Furthermore, we discuss how these challenges can be addressed by ICT-based treatment techniques.

Research efforts regarding biological treatments mainly focus on the following directions:

1) Neuroprotection: Delivering drugs onto the injury site is a possible method of neuroprotection. Recent research in this area concentrates on drug delivery mediated by nanomaterials (e.g., nanowires, nanoparticles, and carbon nanofibers), which have several advantages over conventional drug delivery methods, such as providing targeted delivery and reaching injury site by crossing the blood-spinal cord barrier [50]. The challenges in this direction include the following:

- Application: Devastating effects of secondary injury mechanisms spread fast with the cascade of biological complex events. Preventing this spread means intervening many biological processes in the spinal cord with presumably number of different neuroprotective agents. Thus, identifying the effective drugs and their administrations in a limited time period poses challenges. Moreover, there are many issues regarding drug carrier design [50].
- Validation: Several drugs are tested on animals and in vitro environments for different injury scenarios. Identifying effective drugs and methods among many alternatives and verifying the efficacy by human trials still pose a challenge.

- Functional outcome: Although several neuroprotective drugs are reported to be resulting in some functional recovery [51], functional outcome is limited because complete recovery also necessitates the reformation of spinal cord circuitry.

2) Neural Regeneration: Delivery of neuro-regenerative drugs, cell-based therapy and tissue engineering aim to promote neural regeneration. In drug delivery, growth factors, such as neurotrophins, help in improvement of nerve regeneration, synaptic transmission, and plasticity [52]. Cell therapy efforts mostly focus on cell transplantation and grafting. Transplantation of stem cells is widely investigated as a cellbased therapy [53]-[55] since stem cells have differentiation capabilities that allow them to change into neurons and glia. In addition, a variety of cells are used in studies, such as Schwann cells, that are in-charge of producing myelin sheaths in peripheral nervous system and capable of improving remyelination as well as axonal regrowth [56], [57]. Tissue engineering aims to promote regeneration of injured nerves by means of materials that can mimic natural scaffolding, such as hydrogels, nanofiber scaffolds, and hybrid applications. Hydrogels are biocompatible networks of polymers that can improve the environment for neural regrowth [46], [58], [59]. Nanofiber scaffolds not only provide supportive scaffolding for damaged cells but also deliver drugs and support transplanted cells [60]-[62]. Various combinations of hydrogels, nanofibers, and drugs are also studied for recovery of SCI as shown by $[63]-[65]$. Fundamental challenges in this direction include the following:

- Functional outcome: Although the above-mentioned studies report some improvement regarding neural regeneration, and recent research show several initial results regarding functional neural network formation [66]-[69], directing regenerating neurons to form functional circuitry is still challenging [70].

- Human Trials: Safety and efficiency of medicine treatment and cell therapy on humans are still controversial [70], [71]. Regarding tissue engineering, functionally integrating biocompatible materials to the tissues and the feasibility of human trials pose potential challenges [72].

\section{E. Biological versus ICT-based Treatments}

Since the ICT-based treatment approaches do not have protective or supportive aims, they directly focus on communication problems between the brain and the spinal cord. Several initial studies have provided promising results for these techniques with regard to safety and the functional outcome. In this respect, some advantages over biological treatment approaches are stated below:

- Functional outcome: As processing, stimulation, and communication capabilities of the external interfaces advance with the developments in ICT, restoration in the movements of a subject with SCI can be observed as proved by a recent study [24]. 


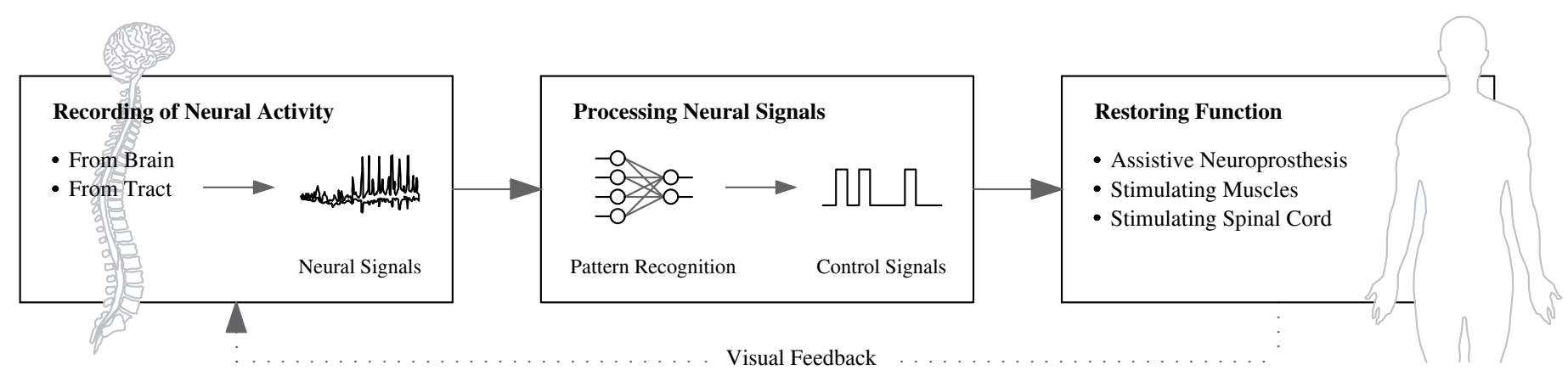

Fig. 3: Basic structure of an NIS, which restores the motor function by using the nervous signals directly captured from the brain or just before the injured part of the spinal cord.

- Human trials: In addition to animal experiments, in studies, such as [25], promising improvements in terms of motor impairment in human patients were observed.

- Safety: Although the long term safety of invasive recording devices in NIS is still not proven, there are less invasive alternatives with low risk neural interfaces, such electroencephalography (EEG)- [73] and electrocorticography (ECoG)-based systems.

\section{Existing ICT-BAsed Treatment TeChniques}

In this section, we present prevalent ICT-based treatment techniques reported in the literature as well as future directions that may be possible in the NIS and AN frameworks. Two techniques contrast with each other on the scale of their approaches with NIS focusing on macro-scale solutions and AN employing a micro/nano-scale approach.

\section{A. Neural Interface Systems}

While the aforementioned biological techniques aim to improve signal transmission problem through the injured spinal cord, NIS utilize nervous signals captured directly from the brain or from the spinal cord before the injured part to restore the motor function. Fig. 3 depicts the major components of a typical NIS, details of each are elaborated below.

1) Nervous recordings: As mentioned, neural signals can be either recorded from the brain [11], [19], [24], [27], [74][76] or the spinal cord [9]. While the former is more common in literature, the latter can potentially provide signals with more information from just a small anatomical region. The recording region is one of important factors that needed to be selected and optimized according to the purpose of NIS. The recording region of the existing experiments in the NISs literature are tabulated in Table II

The key factors in selecting a recording technique in an NIS include efficacy, safety (particularly with regards to surgery), reliability, and longevity. Furthermore, ease of NIS training, support, cost, and availability are also important factors. In the following, we review signal recording techniques from both of the signal sources, and discuss their advantages as well as limitations.

a) Recording from the brain: Brain signals can be recorded via non-invasive techniques, such as EEG, magnetoencephalography (MEG) or functional magnetic-resonance imaging (fMRI). These techniques have low spatio-temporal resolution, low signal-to-noise ratio (SNR), and poor sensitivity to high-frequency changes. Hence, they are insignificant for investigating the short-lived spatio-temporal dynamics of many brain processes [88]. However, the feasibility of these techniques in detecting motor intent to some extent is demonstrated in literature, such as [19], [27], where robotic exoskeletons are operated with use of control signals extracted by EEG and electro-oculography recordings.

In contrast, recordings with exceptionally high SNR, lower sensitivity to artifacts than EEG, and high spatio-temporal resolution can be achieved by invasive recording techniques such as ECoG and intracortically electrode placements [88]. In ECoG, electrodes are implanted subdurally on the surface of the brain; hence, they are able to record the activity of groups of neurons and provide movement-related field potentials. These recordings are robust over long periods [89], and higher spatio-temporal resolutions can be achieved by utilizing the micro-ECoG grids [90]. Furthermore, the ECoG signal is used for offline decoding of seven degrees of freedom for arm movements in monkeys and online decoding for individual finger movements in human subjects [84], [91]. This demonstrates that it can provide enough independent control signals for simple loco-motor task.

However, the implantation of electrodes in the cortex is required to directly record neuronal action or local field potentials from the brain and to increase the spatial resolution of the recordings. This implantation yields to significant clinical risks as a result of infection or the formation of scar tissues. Hence, the electrodes need to be placed on a very small cross sectional area to minimize the damage to the tissue. Moreover, this recording technique requires sophisticated signal processing and computationally intensive algorithms to interpret the neural activity and reliably separate signals of different neurons [92]. Although point-and-click control is shown to be successfully achieved 1000 days after implantation of a 100electrode array in an individual with tetraplegia [15], the longterm stability of the implanted electrodes need to be studied in more depth. In particular, intracortical recordings face short-term recording failures due to implant micro-motion, mechanical mismatch of the device and tissue, foreign-body response, and formation of glial scar tissue that interfere with signal transmission [93]. 
TABLE II: Nervous recordings in the NIS literature.

\begin{tabular}{|c|c|c|c|c|}
\hline Purpose & Recording region & Recording & Subject & Ref. \\
\hline $\begin{array}{l}\text { Self modulation of sensori- } \\
\text { motor EEG rhythms, related } \\
\text { to the imagination of limb } \\
\text { movements }\end{array}$ & Scalp potentials from 59 positions & $\begin{array}{l}\text { Electroencephalography } \\
\text { (EEG) }\end{array}$ & Human & $\overline{77}$ \\
\hline $\begin{array}{l}\text { Advanced wheelchair con- } \\
\text { trol }\end{array}$ & Scalp potentials from 32 positions & EEG & Human & $1 \overline{78}$ \\
\hline Control hand exoskeleton & $\begin{array}{l}\text { EEG from } 5 \text { positions and electro- } \\
\text { oculography recordings (EOG) }\end{array}$ & EEG and EOG & Human & {$[\overline{19}$} \\
\hline 1D cursor movement & $\begin{array}{l}\text { Electrodes placed subdurally on the cor- } \\
\text { tical surface, in some subjects, electrode } \\
\text { coverage included sensorimotor or speech } \\
\text { cortex areas }\end{array}$ & $\begin{array}{l}\text { Electrocorticography } \\
\text { (ECoG) }\end{array}$ & Human & $\overline{\overline{79}}$ \\
\hline Control a 1D cursor & $\begin{array}{l}\text { Left frontal-parietal-temporal region in- } \\
\text { cluding parts of sensorimotor cortex }\end{array}$ & ECoG & Human & $\overline{80}$ \\
\hline $\begin{array}{l}\text { Decoding two-dimensional } \\
\text { movement trajectories }\end{array}$ & $\begin{array}{l}\text { Fronto-parietal-temporal region including } \\
\text { parts of sensorimotor cortex }\end{array}$ & ECoG & Human & $\overline{81}$ \\
\hline Decoding motor intentions & $\begin{array}{l}\text { Hand area of the ipsilesional primary mo- } \\
\text { tor cortex, premotor and somatosensory } \\
\text { areas }\end{array}$ & ECoG & Human & {$[\overline{82}$} \\
\hline $\begin{array}{l}\text { Control of 3D cursor move- } \\
\text { ment }\end{array}$ & $\begin{array}{l}\text { Hand and arm area of the left sensorimotor } \\
\text { cortex }\end{array}$ & ECoG & Human & $\overline{83}$ \\
\hline $\begin{array}{l}\text { Control individual finger } \\
\text { movements in real time }\end{array}$ & sensorimotor regions & ECoG & Human & $\overline{\overline{84}}$ \\
\hline Reaching and grasping & $\begin{array}{l}\text { Dorsal premotor cortex (PMd), supple- } \\
\text { mentary motor area (SMA), the primary } \\
\text { motor cortex (M1), primary somatosen- } \\
\text { sory cortex (S1), the medial intraparietal } \\
\text { area (MIP) of the posterior parietal cortex } \\
\text { (PP) }\end{array}$ & Single unit & Monkey & $\overline{85}$ \\
\hline $\begin{array}{l}\text { One-dimensional } \\
\text { movements of a robot } \\
\text { arm }\end{array}$ & PMd, M1, PP & Single unit & Monkey & $\overline{6}$ \\
\hline A continuous reaching task & M1 & Local field potentials & Monkey & $8 \overline{86}$ \\
\hline Control a 2D cursor & M1, PMD & Local field potentials & Monkey & 87 \\
\hline $\begin{array}{l}\text { Long-term control of cursor } \\
\text { trajectory and click }\end{array}$ & Arm area of motor cortex & $\begin{array}{l}\text { Single unit and local field } \\
\text { potentials }\end{array}$ & Human & $\overline{15}$ \\
\hline $\begin{array}{l}\text { Restoring weight-bearing } \\
\text { locomotion of the paralyzed } \\
\text { leg }\end{array}$ & Leg area of motor cortex & $\begin{array}{l}\text { Spiking activity of neu- } \\
\text { ronal ensembles }\end{array}$ & Monkey & $\overline{24}$ \\
\hline Encoding of forelimb forces & Corticospinal tract (CST) & Recording from spinal tract & Rat & {$[\overline{9}$} \\
\hline $\begin{array}{l}\text { Prediction of forelimb mus- } \\
\text { cle EMGs }\end{array}$ & CST & Recording from spinal tract & Rat & {$[10]$} \\
\hline
\end{tabular}

b) Recording from spinal tracts: Although recording electrical signals from the brain is largely studied in the literature, many technical challenges still exist in the use of microelectrodes to reach a stable recording of individual cell activities. The most important one is the formation of a layer of activated astrocytes around the recording electrode that makes long-term recording of single spikes very difficult. Hence, recording motor control signals through cross-sectional area of corticospinal tract is investigated in [9], [10]. The main drawbacks of using this recording source for NIS can be listed as the following:

- Designing a mechanically stable array of electrodes is challenging.

- The range of signal capture by an electrode array is limited, and thus the number of neural sources are far more than the array recording electrode contacts.

- The control signal cannot be received in corticospinal tract for patients with tetraplegia or ALS.

2) Signal Processing: Real-time processing of the recorded neural data needs to be done for deriving the intended task by the patient. This process is seen as a pattern recognition system that contains three main parts: (i) feature extraction; (ii) dimension reduction and feature selection; (iii) classification or regression. First, the data is processed to derive a set of features. Feature sets extract the discriminating information that represent a dataset. Since the brain signals are the combination of several simultaneous sources and noise, extraction of an appropriate feature set is a challenging task. The data can contain undesirable components, i.e., artifacts, which need to be removed to improve the performance of NIS. Moreover, not all of the recorded signals through multiple channels are relevant for understanding the phenomena of interest. Hence, dimension reduction and feature selection methods are utilized to remove irrelevant and redundant information. In Table III, a 
TABLE III: A list of methods used in a pattern recognition system.

\begin{tabular}{|c|c|c|}
\hline Process & Method & Ref. \\
\hline \multirow{4}{*}{ Feature extraction } & AutoRegressive component & 94 \\
\hline & Wavelet transform & $\overline{\overline{95}}$ \\
\hline & Common spatial pattern & [96, \\
\hline & Matched filtering & [98] \\
\hline \multirow{2}{*}{ Dimension reduction } & Principal component analysis & [97, ,99] \\
\hline & Independent component analysis & [97], [99] \\
\hline \multirow{2}{*}{ Feature selection } & Genetic algorithm & [99] \\
\hline & Sequential selection & {$[100]$} \\
\hline \multirow{5}{*}{ Classification } & Linear discriminant analysis & 97, 99 \\
\hline & Support vector machine & [97], 99$]$ \\
\hline & Bayesian statistical classifier & {$[96],[101]$} \\
\hline & K-nearest neighbor classifier & [97], [101] \\
\hline & Artificial neural network & [102] \\
\hline
\end{tabular}

list of methods available for each step of a pattern recognition system is provided [100].

Apart from the aforementioned methods, a deep neural network (DNN), which can be trained by a training set of recordings, can also be utilized to extract the task intended by a patient. The advantage of using a DNN is that it extracts the features by itself, which is a complex task as the recorded neural data are high-dimensional and not wellknown. Furthermore, the DNN predicts the non-linear systems more accurately [103].

After extracting the intended task from neural recordings, control signals must be derived to restore or replace natural function of a paralyzed or lost limb. In case of spinal cord stimulations for restoring the functionality of the injured spinal cord, these control signals are the parameters needed as the input of the spinal cord stimulator. In [24], the extensor and flexor hotspots corresponding to different brain signals are identified in intact monkeys, then the monkeys are paralyzed. Since such training data is not available in paralyzed patients, experiments on healthy subjects are needed to detect whether these hotspots are almost the same in different subjects. Even if these hotspots are placed in different locations for each subject, the epidural stimulation method of [26] together with a machine learning (ML) algorithm can be used to gather the recorded data from healthy subjects and choose stimulation parameters [26].

3) Restoration of Function: The extracted control signals from the previous steps are then utilized to restore or replace natural function of a paralyzed or lost limb. Such NIS include brain-computer/machine/spine interfaces [7], [8], [12]-[25], [27], [100], or spinal cord-computer interfaces [9], [10] that are described below:

a) Operating assistive neuroprosthesis: Real-time control of robotic prosthesis and exoskeleton finds its applications in practical rehabilitation by replacing the lost motor function to support of daily actions. Studies on using intra-cortical recordings of the brain to control robotic prosthesis [16]-[18] demonstrate that paralyzed patients may recreate multidimensional control of complex devices even years after the injury. This is utilized in [19] to control a hand exoskeleton that helps patients in restoration of independent daily life activities.
Moreover, with sufficient trainings and use of EEG-controlled robotic actuators, it is shown that patients achieve the ability to perform voluntary motor control in key muscles below the spinal cord injury level [27]. However, using assistive neuroprosthesis is not the focus of this study since it does not provide a solution for bypassing the injured part of the spinal cord to restore the control over muscles.

b) Direct stimulation of muscles: Direct stimulation of muscles by signals extracted from intra-cortical recordings was first studied on monkeys with a transiently paralyzed arm [20]-[22], and then applied to a paralyzed human [25]. In both studies, the exact control signals for each of the muscles required in an activation are extracted and then applied to stimulate the muscles. Results suggest that intra-cortical recordings can be linked to muscle activation in real-time, enabling the control of muscles using the activity of neurons in the motor cortex. This process essentially bypasses the spinal cord and restores the voluntary control of the paralyzed muscles. However, electrical stimulation of muscles may cause fatigue, and long term stimulation by electrodes can damage muscles.

c) Spinal cord stimulation: Epidural spinal cord stimulation and intraspinal microstimulation are two existing methods in the literature for inserting motor control signals back to the motor circuitries. In the first one, the stimulating electrodes are placed in the epidural space of the spinal cord [26], while the electrodes are implanted within the ventral gray matter [104], [105] in the second method.

The sensory feedback from the body, which is processed by neurons in the spinal cord, is crucial in controlling the motor commands [107]. Hence, the idea of stimulating the spinal cord just enough to make it sensitive and responsive to the sensory input is studied in [26]. An array of 16 electrodes is implanted in epidural space of the spinal cord of an individual with a clinically complete motor injury. These electrodes are placed over the identified extensor and flexor hotspots, and a pulse generator is used to control the stimulation parameters. Different set of stimulating parameters are then sent to the pulse generator to observe the response of the patients body to the stimulation. The epidural stimulation in [26] resulted in locomotor-like patterns and full weight-bearing standing with assistance provided only for balance. Instead of selection of stimulating parameters by a physician, which is done in [26], the recorded signals from brain or spinal cord can be processed by a processing unit to set these parameters. Using brain signals to control the epidural stimulation is studied in [24], [108], where locomotor movements, namely walking and climbing, are achieved in paralyzed monkey and rat, respectively.

In [23], intra-spinal micro-stimulation is used to control hand movement in monkeys whose hands were temporarily paralyzed. This stimulation method provides more natural recruitment order of motor units and reduces the number of required electrodes and controllers compared to epidural stimulation. However, higher risk of tissue damage exists due to implantation of electrode into the spinal tissue. Implanted electrodes retain a scar tissue, and long-term stability and safety of the implanted electrodes are some of the major 


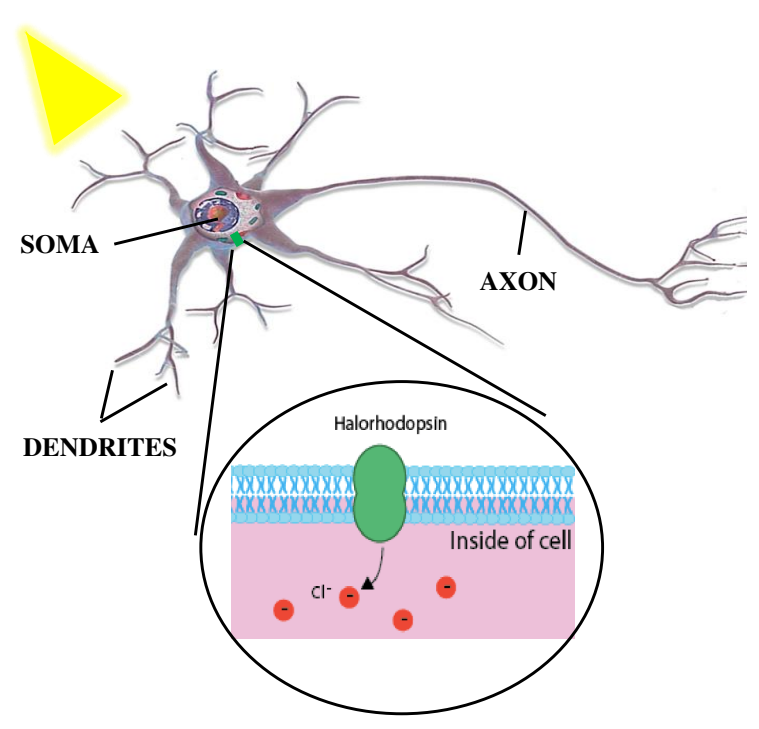

(a) Yellow light activates the opsin called halorodopsin turning the neuron off.

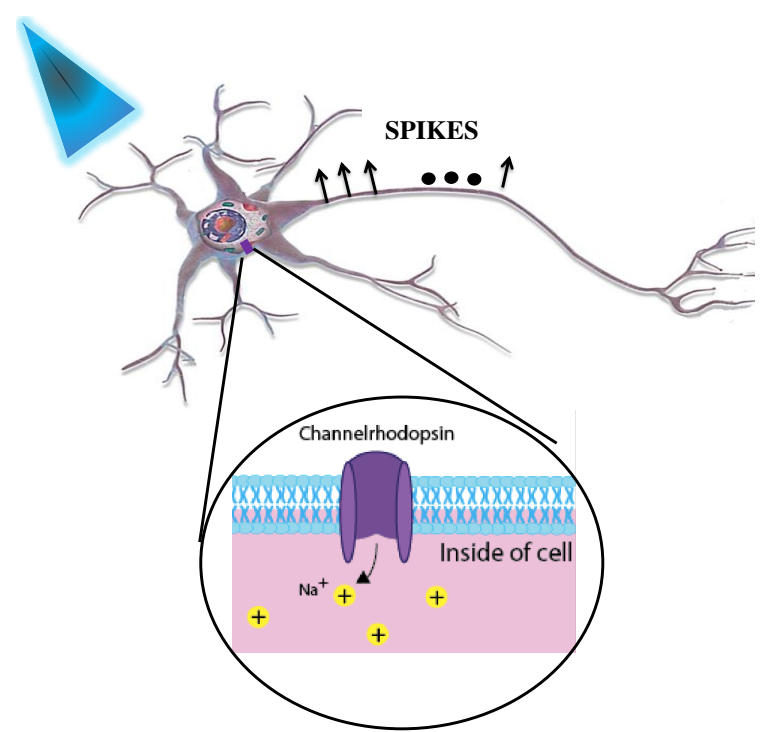

(b) Blue light activates the opsin called channelrhodopsin turning the neuron on.

Fig. 4: Optogenetic neuronal inhibition and excitation using light responsive proteins called opsin [106].

challenges [109].

By using current electrical stimulation techniques in either epidural stimulation or intra-spinal micro-stimulation, cellspecific activation of spinal cord's motor circuitry is challenging due to interference from non-target neuron populations. In this respect, optogenetics can be a key to control the limbs by activating or suppressing the specific population of neurons in the spinal cord as shown in Fig. 4 [110], [111]. Open issues include finding encoding methods for motor commands recorded from the brain in order to deliver meaningful signals to the spinal cord and developing proper light delivery techniques for spinal cord [112]. The former requires identifying contributions of different neuron populations on the motor control. Regarding the latter, current research focuses on the RF-powered wireless systems [113], [114] since employing optical fibers in the spinal cord is not possible. This is contrary to the case of the brain since penetrating an optical fiber in the spinal cord requires the severing of white matter tracts, which carry high information density and have minimal redundancy; thus, local damage can have global consequences [112]. Another approach could be designing an ultrasoundpowered optogenetic stimulator system, such as those in [115], [116], which can reach deeper layers in the spinal cord, to employ them in the spinal cord.

\section{B. Artificial Neurons}

Another potential direction for the treatment of SCI could be the use of artificially-created neurons to replace the injured biological neurons. If perfectly implemented, such an approach can potentially reverse the loss of any function occurred due to SCI; however, the associated challenges are not negligible by any means either. The general architecture of an artificial neuron (AN) is shown in Fig. 55, where a pre-synaptic neuron connects to a processing unit by means of a transducer.
The AN performs processing actions similar to its biological counterparts, and thus completes the nervous pathways.

Some key characteristics required by a functioning AN that may replace biological neurons are outlined below:

- Plasticity: The AN should be able to change synaptic weights in a plastic manner since plasticity forms the basis of memory and cognitive functions of the nervous system and is observed in all neurons in a nervous system. It equips a network of ANs with the capability of mimicking the observed behavior in networks of biological neurons of changing activity patterns according to durable change in input stimuli [117].

- Implementation scale: The scale of implementation decides the level on which the system behaves similar to a biological neuron. From the perspective of a single neuron, the scale can either be synaptic or neuronal. On the synaptic scale, individual synapses can be identified and interfaced to synapses from other neurons, whereas on the neuronal scale, the neuron as a whole may be interfaced. Both systems require a different set of interfacing techniques but the complexity and control available in AN systems will increase against a decrease in the scale.

- Biocompatibility: Same as NIS, the AN needs to provide lifelong stability, which requires biocompatibility and cytocompatibility of the device, i.e., the device must integrate properly within the body and neural tissues, and provide the prolonged maintenance of the desired performance [118].

- Physical dimensions: The size of ANs need to be defined based on the biological neurons that they will be integrated with. Thus, their dimensions must have parts on the scale of micrometers to be compatible with thicknesses of axons and dendrites of biological neurons, which need to be integrated together with other parts of an AN of larger dimensions.

- Energy requirements: Energy self-reliance is another major 


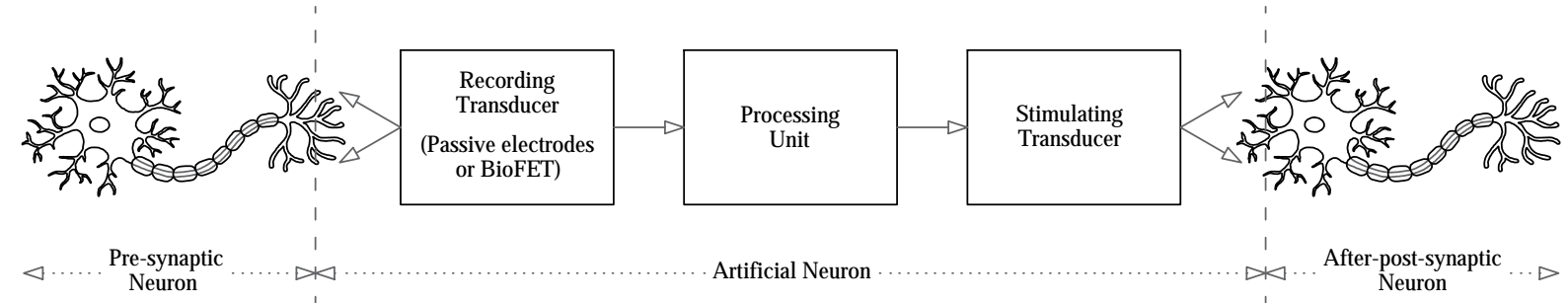

Fig. 5: The general architecture of an AN, which fulfils processing tasks similar to its biological counterparts, completing the nervous pathways.

issue for any implanted system. Use of batteries may not be a viable solution for long-term operation. The energy required by an $\mathrm{AN}$ can be harvested from the body heat or blood pressure by creating gradients within the body [119]; however, other novel methods can be proposed. For instance, ANs may be designed such that they can harvest energy directly from the energy sources of the body, such as glucose, and their efficiency should also be considered.

- Amplification: Processing of biological inputs may require the use of amplifications since the transmission needs to be carried over some distance and the typical energy of signals is on the order of $\mu \mathrm{J}$. Such amplifiers must have low noise, low power, and high precision characteristics.

- Implanting process: This process is almost as critical as the development of ANs. Considering the scale of neurons, operation by a human surgeon is not a viable option. The implant technologies may require the use of robotic surgeries that are capable of operating at micrometer scales, which is already a realized technology by Neuralink [120] for the case of neural electrode implantation. In such cases, the system should be intelligent since the sheer number of decisions will be too large for a human to process. The system would need to automatically identify different sections of biological neuron by means of imaging, and proceed with the surgery by implanting the appropriate AN sections accordingly. Thus, apart from the robotic arms and surgery tools, the implantation requires significant ML and artificial intelligence (AI) systems.

The implantation problems elaborated above can be mitigated by using systems that deploy themselves by means of self-organization.

The characteristics/requirements of ANs pose significant challenges; however, once developed, the benefits of such systems are also unlimited. Apart from the treatment of SCI, they may be utilized to develop computing systems similar to the human brain. Additionally, they may be used as bio-cyber interfaces for applications targeting the nervous system.

To date, there is no working prototype of an AN that may be considered as a complete replacement for a biological neuron. Thus, we discuss some major studies that are approaching to the problem in a similar manner. Two major directions reported, namely neuromorphic and biomimetic neurons, are explained below:

1) Neuromorphic neurons: Neuromorphic neurons are based on electronic circuits, which particularly perform neuromorphic computation, where very-large-scale integration
(VLSI) of individual neurons or synapses are utilized in a similar manner to the human brain [121].

The biggest advantage of neuromorphic systems is that they include plasticity as the basis of their models; however, implementation of ANs by neuromorphic methods faces several bottlenecks, such as huge size, high-power requirements, and interfacing issues. Even for some of the newer devices that use low-power and have small sizes, interfacing issues remain since neuromorphic systems are not designed with neural interfaces [122].

2) Biomimetic neurons: Biomimetic circuits mimic biochemical processes using synthetic materials [123]. Since most communication between biological entities occurs by means of molecule exchange and biochemical reactions of these molecules, biomimetic circuits are ideal candidates for direct interfacing with biological systems. They are employed to realize applications, such as artificial muscles, smart membranes, biological transducers, and brain-machine interfaces [123].

The basic function of a neuron is realized by means of enzyme-based amperometric biosensors and organic ion pumps in a biomimetic fashion by [124]. Although this work is bio-compatible and the detection of bio-molecules is achieved on the scales similar to those of biological neurons, many of the characteristics expected from an AN are not met. For instance, there is no plasticity in the system, macro-scale dimensions are used [124], and the system is tested in vitro without any interface to the biological neurons.

Similar studies that include plasticity and direct interface with biological neurons may be the first step towards the development of organic biomimetic ANs. Size reduction and in vivo testing may follow afterwards. Another direction could be the use of plasticity designs from neuromorphic systems based on biomimetic implementations. This may be the approach that is most successful and has the least time-to-market.

\section{Proposed Approaches of the ICT-Based TREATMENT OF SCI}

In this section, we introduce two potential approaches for the treatment of SCI by considering the current literature and the desired characteristics of NIS and ANs.

\section{A. Neural Interface Systems with Enhanced Feedback}

The main technical challenges in using an NIS to enable the ICT-based treatment of SCI are over-viewed below: 
- Providing somatosensory feedback: It is shown that visual monitoring of a limb's motion can partially correct the shortcomings in movement of patients with large-fiber sensory neuropathy. These subjects face slow and uncoordinated movement as a result of the somatosensory feedback for normal motor control [125]. Hence, patient's vision provides a feedback to the NIS. However, the effect of visual feedback on movements is not as fast as proprioceptive system, which then causes instability in movement [126]. Thus, utilizing proprioceptive feedback or a feedback from spatio-temporal characteristics of limb's in an NIS can assist patients to perform more natural movements and also feel stimulated limbs or neuroprosthesis [108], [127]. As an example, stimulating peripheral afferent nerves in the limb's stump with intrafascicular electrodes [128], [129] or cuff-like electrodes [130] is studied in the literature for providing the natural sensation of missing limbs in amputee subjects. However, these methods are not applicable in paralyzed patients, who have also lost the sensory pathway. In such cases, the tactile and proprioceptive feedback must be provided by artificially stimulating the sensory cortex.

- Appropriate neural recording technique, spinal cord, and brain stimulation: Lifelong stable neural signal recordings and stimulation are required for which the important factors are reliable chronic recordings, biocompatibility and cytocompatibility of the device, the ability of the device to integrate properly within neural tissues, and the prolonged maintenance of desired electrical properties. Furthermore, both recording and stimulating devices must have high spatio-temporal resolution to handle the required degree of freedom for restoring function after the SCI. Graphene is a bio-compatible 2D material, and its sensitivity to different ions can be modified by various etching methods and functionalization [131]. Hence, it can be used for sensing fluctuations in specific ion concentrations. Graphene is electrically conductive, and it can be patterned into nanoscale, electrically disconnected, conductive patches via hydrogenation, etching or fluorination [132], [133]. This makes it suitable for implementing high resolution neural interfaces which can both generate and differentiate the highly localized fluctuations in ion concentrations.

- Fully implantable system: To offer more comfort for patients and reduce the risk of inflammation, the NIS must be fully implantable. Moreover, the system must provide the required bandwidth for transmitting data between its different components while considering the power consumption.

To address the above-mentioned challenges, we propose a comprehensive framework for realizing an NIS with enhanced feedback, EF-NIS, that utilizes the neural signals recorded from the brain and stimulates the spinal cord for restoring motor functions in the paralyzed patients as shown in Fig. 6 . Components of the proposed EF-NIS are explained below:

1) Recording device and the communication interface: Graphene-based devices are particularly attractive for neural interfacing as graphene promotes neurite growth [134], do not alter the behavior of target neurons [135], is flexible and transparent, which allows neuroimaging/optogenetic stimula- tion concurrent to neural electrophysiology [136]. Moreover, graphene is highly conductive and electrically sensitive, ideal for lowering SINR [137]. To this aim two set of devices are studied in the literature. Graphene based Micro-Electrode Arrays (MEAs) provide very dense arrays with submicron electrodes [138], [139] while having easy and reliable fabrication process. However, MEAs do not provide any on-site amplification thus implying noise in links severely effects SINR and the recorded signal needs complicated post processing. On the other hand, graphene Field Effect Transistors (GFETs) provide on-site amplification of the recoded signal, thus, high SINR values [140]. In addition, biochemical sensing of neurotransmitters, e.g., glutamates, is possible by functionalization of the surface [141]. By selecting the appropriate receptor molecules, we can mimic realistic postsynaptic membrane potential decay times, which leads to implementation of both temporal and spatial summation of postsynaptic membrane potential. Thus, the graphene-based high resolution neural interface (GNI) is suggested to record the brain activities of the patient from primary motor cortex. This recording device is implanted together with a processing unit and a transmitter, which reports the recorded signals in a wireless manner.

The bandwidth demand of the wireless transmission channel between the brain implant and the external processing unit is correlated with the number of recording channels employed. For better estimation of brain commands, we need densely distributed recording sites that require high data rates (on the order of Mbps) [142]. On the other hand, the transmitter should dissipate low energy because even $1^{\circ} \mathrm{C}$ of temperature increase can damage neural tissues [143]. Considering these limitations, ultra-wide band (UWB: $3.1-10.6 \mathrm{GHz}$ ) systems, which can provide high data rate within allowable power dissipation range with the minimal chip area [144], is the most suitable solution [145]. Therefore, the communication interface between the brain implant-processing unit of the EFNIS consists of a simple UWB transmitter at the brain implant and a more complex UWB receiver at the processing unit [146]. Furthermore, compression techniques with efficient implementation, similar to one in [147], can be utilized to reduce data rate without considerably increasing the complexity.

2) Processing unit and the spinal stimulator interface: The processing unit performs two tasks: (i) decoding the intent of the subject from neural signals; (ii) generating control signals for spinal cord stimulation. This unit will be placed in a rechargeable and mobile device. Hence, it needs a wireless transmitter to transfer the stimulation parameters to the site of spinal cord stimulation. The transmission between the processing unit and the spinal cord neural stimulator can be performed at lower data rates compared to the brain implant-to-processing unit link since the dimensionality of the transmitted signal reduces. Additionally, the receiver at the neural stimulator must operate with low energy [148]. Areaand power-efficient UWB transmission is suitable for this link [149], [150]. Moreover, it is also advantageous in terms of interference [151].

The received control signals at the spinal cord stimulator are then used for setting the stimulation parameters. The GNI is able to both recognize and generate fluctuations in 


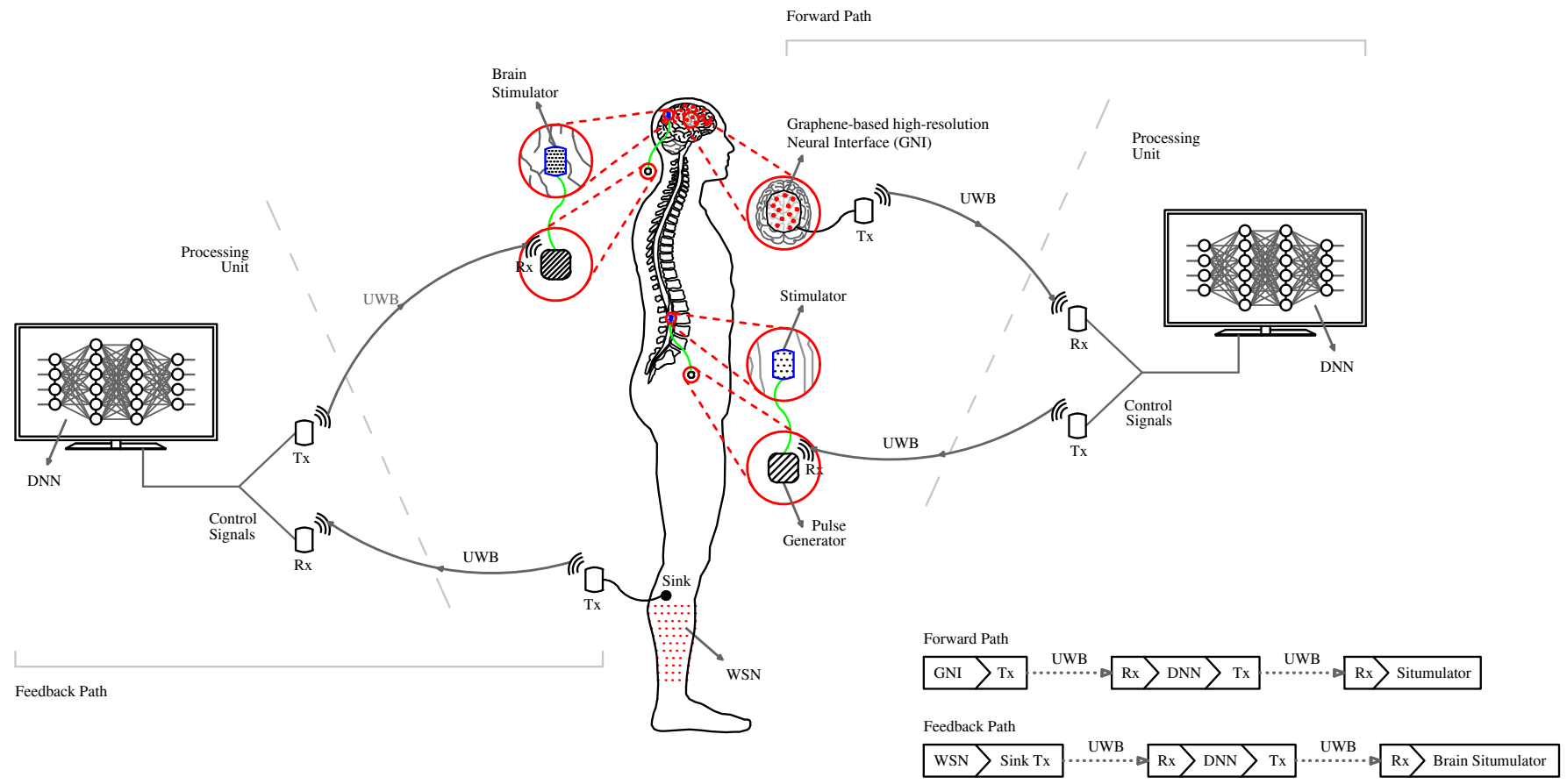

Fig. 6: The neural interface system with enhanced feedback, EF-NIS, that utilizes brain-recorded neural signals and stimulates the spinal cord to restore the motor functions of the paralyzed patients.

concentration of specific molecules in a local area. Hence, it will also be used for high resolution stimulating of nerve cells located in the spinal cord.

3) Muscle to brain communication interface (Feedback): In literature, there is a variety of wearable sensors for monitoring the body activity. Among those, especially flexible tattoobased and skin-like sensors are profitable due to their soft and ultra-thin designs that provide comfort for users. Furthermore, they are generally made of bicompatible materials, such as graphene [152]-[155] and gold [156], as shown in Fig. 7 , which makes them suitable for long-term use. These sensors are also capable of recording multiple sensory modalities, such as muscle activity and touch [152]. For the communication interface, wired, wireless or hybrid approaches can be followed. For high density of sensors, which is the case of multiple modalities being recorded, we can use wired sensor arrays, whose recordings are gathered at the sink node, which has a direct wireless communication interface with the processing unit or with the brain implant. Otherwise, communication modules of the sensors can be integrated to Near Field Communication (NFC) and Radio Frequency Identification (RFID) technologies, which are enabled by flexible thin antennas, such as graphene-based antenna shown in [157].

An alternative to wearable sensors is ultrasound-based implantable neural dust, which is validated in rat peripheral nerves and is shown to be capable of recording neural activity in the peripheral nervous system [158]. This system uses backscattering and other advantageous aspects of ultrasound, such as low attenuation through the tissues and low energy consumption compared to RF technologies currently employed in implantable devices [159]. In addition, further miniaturization is needed in this system similar to the aforementioned flexible sensor-based systems.

The collected sensory information is then transmitted to the processing unit to generate the parameters for somatosensory cortex stimulation. For this aim, the responsible regions of somatosensory cortex for sensory feedback from each leg should be identified. This task can be achieved by collecting tactile and muscle activities in healthy subjects, and recording the corresponding activities in somatosensory region during walking. Then, a DNN is trained to estimate the activity of the somatosensory cortex according to the collected sensory information. Since we consider the SCI injuries, in which the sensory pathway is also damaged, EF-NIS utilizes this information to generate stimulation parameters that activate similar somatosensory regions in patients.

In the next step, the processing unit transmits the stimulation parameters to the controller unit that is surgically placed over the pia mater, i.e., the delicate innermost layer of the meninges that is the membranes surrounding the brain and the spinal cord. The transmission is again performed through the UWB communication interface. The controller sends the control signals to the graphene-based high-resolution neural interface, located over the somatosensory region, to stimulate the brain through manipulating ionic concentration levels. A possible approach to achieve the manipulation of ionic concentration levels, graphene membranes with tunable sub-nanometer pores are a suitable candidate, which are shown to help to facilitate selective ion transport through them [160].

\section{B. Self-Organizing Artificial Neurons}

Although the preceding neural interface is relatively easy to implement and may return a high degree of motion to the patients, the movements will not be precise. In similar, 


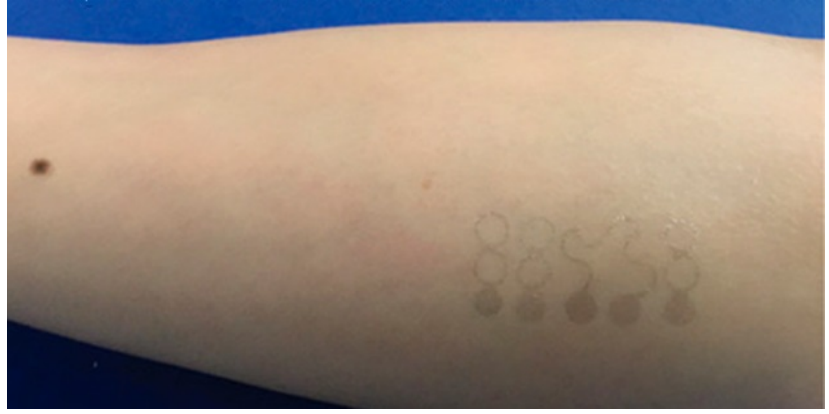

(a) Graphen electronic tattoo sensors 152 .

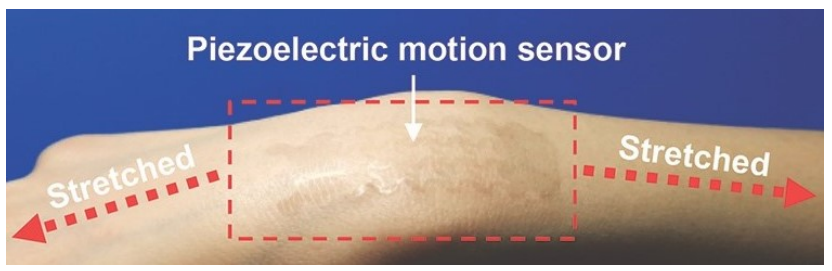

(b) A transparent graphene-based piezoelectric motion sensor [153].

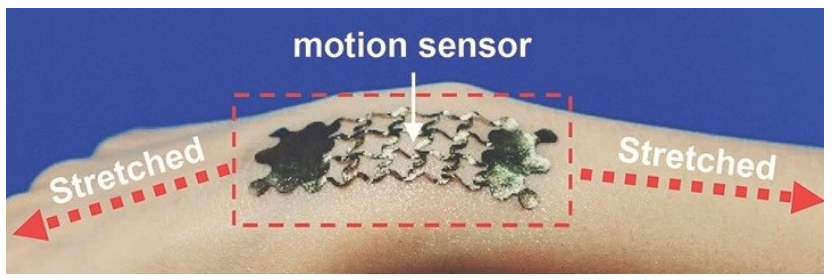

(c) Flexible motion sensor using gold thin films [153].

Fig. 7: Examples of flexible tattoo-based and skin-like sensors.

although the nervous system's information processing capacity increases by the use of an NIS, its communication rate is not as high as before the injury. Before an SCI, being normally wired, the nervous system can be assumed to operate at optimum connectivity with maximum communication rate. However, after the SCI, while using an NIS, neural information can only be communicated at limited neural regions, a setup far from the optimum connectivity, which results in diminished communication rate. This materializes itself, for instance, as limited stimulation of some estimated areas of the spinal cord for the motor movement. Apart from limitations in establishing connectivity, operating through interfaces located far away, e.g., the cerebral cortex, from the spinal injury site, where the lost connectivity to be reestablished resides, additionally suffers from the delocalization of neural signals as they propagate. Thus, establishing a simple lost connection at the injury site may correspond to collecting readings from the multiple sites of cortex together with some signal post-processing. However, the delocalization process, coupled with a lack of complete neural signals, results in unrecoverable information loss, and the lost connection will not be recovered in full effect. On the other hand, delocalization of neural signals is not an issue if one opts to work at the injury site.

A solution to this problem could be the retrieval of connections lost at the injury site via the self-organizing ANs. The connectivity, and consequently communication rates, could be (at least theoretically) brought back to the pre-injury levels by employing artificial nervous connections across the injury site, where the signals are already localized in specific positions. An architecture, where ANs with neuromorphic properties are suspended in a hydrogel, could be employed for such purpose. The hydrogel-suspended network of ANs can form bridges across the injury site and make new nervous connections with biological and artificial neurons via self-organization. A representative diagram is shown in Fig. 8 . Below, we discuss the major properties of the proposed self-organizing AN:

1) Plasticity: In order for an AN to adapt to its environment, plasticity should be introduced in the system and realized by utilizing neuromorphic designs. The firing rate of each AN depends on the signals it receives from synapses with other neurons that may be both biological or artificial. Each synapse of the AN is associated to a variable Weight that models plasticity of the synapse and retains the weight of a particular synapse [161]. The Weight may function to strengthen or weaken depending on the synaptic connection type (excitatory or inhibitory). The overall effect of plasticity is the introduction of memory in the system, which is a key characteristic of the nervous system.

2) Self-organization: Since functional ANs are required to interface with biological neurons, their dimensions are dictated by the task. If ANs are communicating on axonal and somatic levels, their sizes will be micro-scale whereas if ANs aim to communicate on synaptic scales, their sizes should be nanoscale. As discussed earlier, the implantation of AN systems propose a major challenge since surgery on the micro/nanoscale itself is an open problem. This can be bypassed by considering systems that use self-organization to create the AN bridge across the nervous injury. Such a system is shown in Fig. 8, where AN bridges form across the injury site over time.

Self-organization is a hot research area, where local interactions between individual entities give rise to the overall structure of the system. The simplest approach towards this goal is the use of electric or magnetic fields. Since the action potentials of the nervous system are themselves electrical signals, self-organization can be based on its use as the attractor field. The concept of alignment of microstructures by means of anisotropic electric field potentials has already been demonstrated for the case of metallic microcoils suspended in PDMS [162]. Graphene nanosolenoids discussed in [163] may provide the basis for an implementation in this regard, both in terms of the required size and low energy requirements and magnetic properties. The nanosolenoids, however, are not sufficient by themselves for self-organization, because the nervous signals are not continuous in nature and the AN bridge may fall apart if a signal is absent for some time. To ensure long-term endurance of the structures, we either need mechanical structures, such as hinges [164], or the use of magnetic fields external to the body [165].

3) Energy Harvesting: For the system to be completely independent, ANs need to extract energy from their vicinity. This energy can be scavenged within the body [119] or delivered through external sources using wireless power transfer (WPT) [166].

Energy harvesting (EH) within the body can either be based 


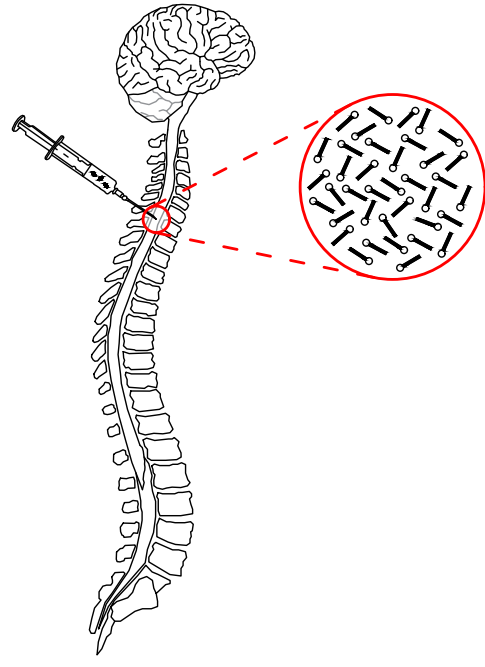

(a) Before self-organization.

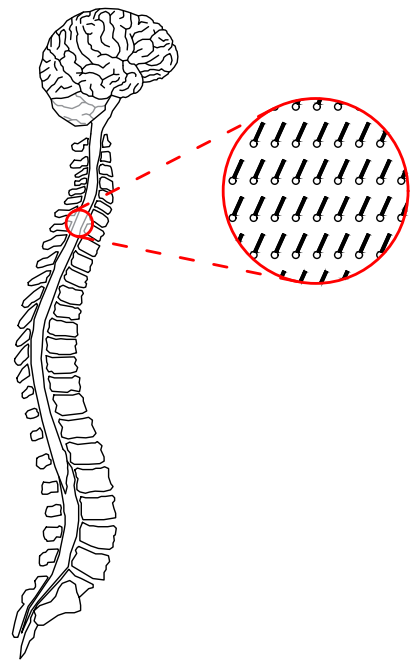

(b) After self-organization.

Fig. 8: Diagram of the self-organizing ANs suspended in a hydrogel, where AN bridges form across the injury site over time.

on biochemical or biomechanical processes [167]. Chemical reactions within the body, such as glucose uptake and food digestion, can be considered as the sources for biochemical EH mechanisms. Here, glucose and oxygen come to the forefront and are used extensively compared to several other biochemical pairs due to their large quantity and ubiquity in human body [168]. When the glucose is burnt with oxygen in an implantable enzymatic biofuel cell, the free electrons are released, which generates the electrical energy. Typical to any chemical reaction, the rate of this oxidation can be increased by such substances called catalysts, the characteristics of which affect the level of power generation. The maximum power density that can be gathered by glucose oxidation is $180 \mu \mathrm{W} / \mathrm{cm}^{2}$ [167].

On the other hand, biomechanical EH can either be based on voluntary body functions, such as movement, or involuntary functions, such as blood flow, breathing, and heartbeat, which create utilizable vibration. The sources of locomotion, i.e., any voluntary movement, can be converted into electricity using piezoelectric and electrostatic materials or nanogenerators. Piezoelectrics can be also used for utilizing the involuntary body functions in addition to nanowires and electromagnetic induction. It is possible to create up to $6 \mu \mathrm{J} / \mathrm{cm}^{3}$ of power density out of blood pressure, which is $1.2 \mu \mathrm{W} / \mathrm{cm}^{2}$ for heartbeat. Depending on the location of sources, hybrid EH mechanisms can be also implemented to increase the likelihood of continuous energy reception, allowing a source to compensate for another in case of failure [169]. The key advantage of $\mathrm{EH}$ within body is the fact that the designed system can be independent of external influences, allowing subjects to move more freely.

WPT can be realized by providing power to the system externally by means or either inductive or magnetic coupling, or by means of electromagnetic radiation [170]. Apart from reducing the challenges of designing $\mathrm{EH}$ mechanisms in an already complex scenario of SCI treatment, if an external source is selected, the same may be used for the self-organization of the AN devices, and as a readout interface of the AN network. Finally, it should be noted that EH is a very active area of research. New efforts are focused on reducing the power requirements of networks by using energy-efficient protocols, conservation schemes, and novel topologies. Future work can further elaborate on the directions overviewed here.

\section{FURTHER APPLiCATIONS OF THE ICT-BASED TREATMENT OF SCI}

By revisiting the current treatment techniques of SCI from an ICT perspective, we can provide rigorous optimization frameworks for the improvement of these techniques. However, apart from SCI treatment, such ICT-based techniques can have applications in a number of other scenarios. Thus, in this section, we discuss some key applications that are realizable as byproducts of ICT-based SCI treatment techniques.

\section{A. The Internet of Bio-Nano Things}

The Internet of Bio-Nano Things (IoBNT) is an emerging subfield of IoT, which focuses on developing artificial biological/bio-inspired nano-scale devices capable of communicating with each other and their surroundings, e.g., biological entities/parts or trace biomolecules from biological entities/parts, to establish predefined tasks at the nano-scale in a biological environment, with envisioned applications ranging from intra-body sensing [171] and actuation networks to environmental control of chemical and biological agents and pollution [172]. In the case of intra-body applications, experience gained from techniques developed for SCI treatments, in particular, molecular NIS, can provide the means for establishing communication with nano-networks deployed into the body. Such applications include remote monitoring of the body for various vectors and automatized targeted drug delivery upon detection of anomalies in observed data. 


\section{B. Neurophysiology/Physiology}

Large amounts of data can be retrieved from the nervous system by using the interfaces of the proposed SCI treatments. These data can be processed to obtain a mapping between the architecture of the underlying network and its function, and contribute vastly to our understanding of biological neural networks. On the other hand, NIS-like systems equipped with molecular sensors capable of sensing specific biomolecules can be used to interface other biological systems, such as cardiovascular and lymphatic systems. Data collected from such an endeavor will significantly contribute to our understanding of human physiology. For instance, we can study nervous-related diseases, such as Alzheimer's disease, multiple sclerosis, and Parkinson's disease, in unprecedented detail using electrical/molecular NIS as interfaces to the central nervous system. This may lead to novel disease diagnosis and treatment techniques as well as the identification of key factors that cause such conditions.

\section{Neuromorphic Computing}

A firm understanding of the relation between the architecture and function of a neural network would allow us to design our own artificial neural networks (ANNs, as a subtopic of ML and $\mathrm{AI}$ ) with specialized functions, and employ these architectures for neuromorphic computation at large scale [173]. Neuromorphic architectures process data in a highly parallel manner as opposed to the serial structure used by von Neumann architectures employed by contemporary computers. This allows ANNs to process data at comparatively very low frequencies, and therefore to be highly energy efficient.

One application of functional ANNs lies in their integration into neural interfaces for on-body preprocessing of collected data, which is necessary as the volume of raw data collected by high spatio-temporal resolution interfaces will be so large that transmission of this data without any preprocessing is doomed to have heating problems, which is not desired for an on-body device. Thus, we will have a positive feedback loop: Understanding gained from neural interfaces will advance our capability of neuromorphic computing, which, in return, will pave the way for more advanced interfaces.

\section{Augmented Humans (Cyborgs)}

The use of external machinery to enhance capabilities of a human body is not new. In fact, prosthetics are widely used to help people with missing limbs to regain some functionalities lost due to suffered injury. With advancements in SCI treatment technology, it is expected that such prostheses will be upgraded from being mere mechanical tools to interactive electronic devices capable of communicating with human nervous system as the so-called neural prostheses [174]. Interesting applications in such directions may include extrasensory feedbacks to achieve new sensations (e.g., augmented reality [175]), mechanical body suits that allow superhuman strength, direct integration of gaming to the human body, among others.

\section{CONCLUSIONS}

SCI are serious health conditions that adversely affect the life of a plethora of patients around the world. Existing biological methods not only aim to re-establish axonal connection by promoting nerve regeneration but also minimize harmful toxicities of the injury mechanisms. However, to date, no existing treatment technique has shown significant success. Among the ICT-based systems, NIS simply bypass the injury site by decoding the brain signals and providing them to the recipient muscles. On the other hand, employment of ANs aims to compensate the functional loss by replacing the injured neurons with engineered neurons. Considering these, we propose two novel approaches, namely EF-NIS (NIS with enhanced feedback) and self-organizing ANs, and identify key areas that may be most important in this regard. For NIS, apart from the need of high resolution recording and stimulation setups, we identify that the feedback from impaired limbs back to the brain is also very essential. For ANs, we propose the use of self-organization to tackle the transplant issues in a smarter way and also EH to make the devices fully independent. The development of these treatments will have a great impact on a number of application areas, such as IoBNT.

\section{REFERENCES}

[1] O. B. Akan et al., "Fundamentals of molecular information and communication science," Proceedings of the IEEE, 2016.

[2] S. G. Waxman, Clinical Neuroanatomy. New York: McGraw-Hill, 2013.

[3] S. L. James et al., "Global, regional, and national burden of traumatic brain injury and spinal cord injury, 1990-2016: a systematic analysis for the global burden of disease study 2016," The Lancet Neurology, vol. 18, no. 1, pp. 56-87, 2019.

[4] "Spinal cord injury (sci) 2016 facts and figures at a glance," The Journal of Spinal Cord Medicine, vol. 39, no. 4, pp. 493-494, 2016, pMID: 27471859. [Online]. Available: http://dx.doi.org/10.1080/ 10790268.2016.1210925

[5] E. E. Fetz, "Real-time control of a robotic arm by neuronal ensembles," Nature neuroscience, vol. 2, pp. 583-584, 1999.

[6] J. Wessberg et al., "Real-time prediction of hand trajectory by ensembles of cortical neurons in primates," Nature, vol. 408, no. 6810, pp. 361-365, 2000.

[7] M. D. Serruya et al., "Brain-machine interface: Instant neural control of a movement signal," Nature, vol. 416, no. 6877, pp. 141-142, 2002.

[8] D. M. Taylor et al., "Direct cortical control of 3d neuroprosthetic devices," Science, vol. 296, no. 5574, pp. 1829-1832, 2002.

[9] Y. Guo et al., "Encoding of forelimb forces by corticospinal tract activity in the rat," Frontiers in neuroscience, vol. 8, 2014

[10] S. Gok and M. Sahin, "Prediction of forelimb muscle emgs from the corticospinal signals in rats," in Engineering in Medicine and Biology Society (EMBC), 2016 IEEE 38th Annual International Conference of the. IEEE, 2016, pp. 2780-2783.

[11] P. R. Kennedy and R. A. Bakay, "Restoration of neural output from a paralyzed patient by a direct brain connection," Neuroreport, vol. 9, no. 8, pp. 1707-1711, 1998

[12] P. R. Kennedy et al., "Direct control of a computer from the human central nervous system," IEEE Transactions on rehabilitation engineering, vol. 8, no. 2, pp. 198-202, 2000.

[13] S. Musallam et al., "Cognitive control signals for neural prosthetics," Science, vol. 305, no. 5681, pp. 258-262, 2004.

[14] L. R. Hochberg et al., "Neuronal ensemble control of prosthetic devices by a human with tetraplegia," Nature, vol. 442, no. 7099, pp. 164-171, 2006.

[15] J. Simeral et al., "Neural control of cursor trajectory and click by a human with tetraplegia 1000 days after implant of an intracortical microelectrode array," Journal of neural engineering, vol. 8, no. 2, p. 025027, 2011. 
[16] L. R. Hochberg et al., "Reach and grasp by people with tetraplegia using a neurally controlled robotic arm," Nature, vol. 485, no. 7398 , pp. 372-375, 2012.

[17] J. L. Collinger et al., "High-performance neuroprosthetic control by an individual with tetraplegia," The Lancet, vol. 381, no. 9866, pp 557-564, 2013.

[18] M. Velliste et al., "Cortical control of a prosthetic arm for self-feeding," Nature, vol. 453, no. 7198, pp. 1098-1101, 2008.

[19] S. Soekadar et al., "Hybrid eeg/eog-based brain/neural hand exoskeleton restores fully independent daily living activities after quadriplegia," Science Robotics, vol. 1, no. 1, p. eaag3296, 2016.

[20] C. T. Moritz et al., "Direct control of paralysed muscles by cortical neurons," Nature, vol. 456, no. 7222, pp. 639-642, 2008.

[21] E. A. Pohlmeyer et al., "Toward the restoration of hand use to a paralyzed monkey: brain-controlled functional electrical stimulation of forearm muscles," PloS one, vol. 4, no. 6, p. e5924, 2009.

[22] C. Ethier et al., "Restoration of grasp following paralysis through braincontrolled stimulation of muscles," Nature, vol. 485, no. 7398, pp. 368-371, 2012.

[23] J. B. Zimmermann and A. Jackson, "Closed-loop control of spinal cord stimulation to restore hand function after paralysis," Frontiers in neuroscience, vol. 8, p. 87, 2014.

[24] M. Capogrosso et al., "A brain-spine interface alleviating gait deficits after spinal cord injury in primates," Nature, vol. 539, no. 7628, pp. 284-288, 2016.

[25] C. E. Bouton et al., "Restoring cortical control of functional movement in a human with quadriplegia," Nature, vol. 533, no. 7602, pp. $247-$ $250,2016$.

[26] S. Harkema et al., "Effect of epidural stimulation of the lumbosacral spinal cord on voluntary movement, standing, and assisted stepping after motor complete paraplegia: a case study," The Lancet, vol. 377, no. 9781, pp. 1938-1947, 2011.

[27] A. R. Donati et al., "Long-term training with a brain-machine interfacebased gait protocol induces partial neurological recovery in paraplegic patients," Scientific reports, vol. 6, 2016.

[28] E. Balevi and O. B. Akan, "A physical channel model for nanoscale neuro-spike communications," IEEE Tran. Com., vol. 61, no. 3, pp. 1178-1187, 2013.

[29] D. Malak and O. Akan, "Communication theoretical understanding of intra-body nervous nanonetworks," IEEE Communications Magazine, vol. 52, no. 4, pp. 129-135, 2014.

[30] D. Malak and O. Akan, "A communication theoretical analysis of synaptic multiple-access channel in hippocampal-cortical neurons," IEEE Tran. Com., vol. 61, no. 6, pp. 2457-2467, 2013.

[31] H. Ramezani and O. B. Akan, "A communication theoretical modeling of axonal propagation in hippocampal pyramidal neurons," IEEE Transactions on NanoBioscience, 2017.

[32] H. Ramezani and O. B. Akan, "Synaptic channel model including effects of spike width variation," in 2nd ACM NANOCOM. ACM, 2015.

[33] H. Ramezani and O. B. Akan, "Importance of vesicle release stochasticity in neuro-spike communication," in 39th IEEE EMBC Conf. IEEE, 2017.

[34] H. Ramezani et al., "Rate region analysis of multi-terminal neuronal nanoscale communication channel," in 17th IEEE NANO Conf. IEEE, 2017

[35] H. Ramezani and O. B. Akan, "Information capacity of vesicle release in neuro-spike communication," IEEE Communications Letters, 2017.

[36] T. Khan et al., "Diffusion-based model for synaptic molecular communication channel," IEEE Transactions on NanoBioscience, 2017.

[37] N. A. Abbasi and O. B. Akan, "A queueing-theoretical delay analysis for intra-body nervous nanonetwork," Nano Com. Net., 2015.

[38] N. A. Abbasi et al., "Controlled information transfer through an in vivo nervous system," Scientific reports, vol. 8, no. 1, p. 2298, 2018.

[39] D. Purves et al., "Neuroscience. 3rd," Massachusetts: Sinauer Associates Inc Publishers, 2004.

[40] M. Civas and O. B. Akan, "Rate of information flow across layered neuro-spike network in the spinal cord," IEEE Transactions on NanoBioscience, 2020.

[41] R. N. Lemon, "Descending pathways in motor control," Annu. Rev. Neurosci., vol. 31, pp. 195-218, 2008.

[42] J. W. McDonald and C. Sadowsky, "Spinal-cord injury," The Lancet, vol. 359, p. 417-425, Feb 2002

[43] S. N. A. et al., "From basics to clinical: A comprehensive review on spinal cord injury," Progress in Neurobiology, vol. 114, p. 25-57, 2014.
[44] R. Papastefanaki, FlorentiaMatsas, "From demyelination to remyelination : The road toward therapies for spinal cord injury," Glia, vol. 63, no. 7, pp. 1101-1125, 2015

[45] C. A. Oyinbo, "Secondary injury mechanisms in traumatic spinal cord injury: a nugget of this multiply cascade." Spinal Cord, vol. 71, pp. 281-299, 2011.

[46] S. Kabu et al., "Drug delivery, cell-based therapies, and tissue engineering approaches for spinal cord injury," Journal of Controlled Release, vol. 219, pp. 141-154, 2015.

[47] A. Llobet Rosell and L. J. Neukomm, "Axon death signalling in wallerian degeneration among species and in disease," Open biology, vol. 9, no. 8, p. 190118, 2019.

[48] A. Prasad and M. Sahin, "Can motor volition be extracted from the spinal cord?" Journal of neuroengineering and rehabilitation, vol. 9, no. 1, p. 41, 2012.

[49] Y.-H. Kim et al., "Spinal cord injury and related clinical trials," Clinics in orthopedic surgery, vol. 9, no. 1, pp. 1-9, 2017.

[50] J. Y. Tyler et al., "Nanomedicine for treating spinal cord injury," Nanoscale, vol. 5, no. 19, pp. 8821-8836, 2013.

[51] A. Faccendini et al., "Nanofiber scaffolds as drug delivery systems to bridge spinal cord injury," Pharmaceuticals, vol. 10, no. 3, p. 63, 2017.

[52] S. M. Willerth and S. E. Sakiyama-Elbert, "Approaches to neural tissue engineering using scaffolds for drug delivery," Advanced drug delivery reviews, vol. 59, no. 4, pp. 325-338, 2007.

[53] P. Lu et al., "Long-distance growth and connectivity of neural stem cells after severe spinal cord injury," Cell, vol. 150, no. 6, pp. 1264 $1273,2012$.

[54] H. Nakajima et al., "Transplantation of mesenchymal stem cells promotes an alternative pathway of macrophage activation and functiona recovery after spinal cord injury," Journal of neurotrauma, vol. 29 , no. 8, pp. 1614-1625, 2012

[55] J. Sharp et al., "Human embryonic stem cell-derived oligodendrocyte progenitor cell transplants improve recovery after cervical spinal cord injury," Stem cells, vol. 28, no. 1, pp. 152-163, 2010.

[56] H. Kanno et al., "Combination of engineered schwann cell grafts to secrete neurotrophin and chondroitinase promotes axonal regeneration and locomotion after spinal cord injury," Journal of Neuroscience, vol. 34, no. 5, pp. 1838-1855, 2014.

[57] D. D. Pearse et al., "Transplantation of schwann cells and/or olfactory ensheathing glia into the contused spinal cord: Survival, migration, axon association, and functional recovery," Glia, vol. 55, no. 9, pp. 976-1000, 2007.

[58] P. Krsko et al., "Length-scale mediated adhesion and directed growth of neural cells by surface-patterned poly (ethylene glycol) hydrogels," Biomaterials, vol. 30, no. 5, pp. 721-729, 2009.

[59] M. J. Mahoney and K. S. Anseth, "Three-dimensional growth and function of neural tissue in degradable polyethylene glycol hydrogels," Biomaterials, vol. 27, no. 10, pp. 2265-2274, 2006.

[60] Y. Liu et al., "A self-assembling peptide reduces glial scarring, attenuates post-traumatic inflammation and promotes neurological recovery following spinal cord injury," Acta biomaterialia, vol. 9, no. 9, pp. 8075-8088, 2013.

[61] J. Guo et al., "Reknitting the injured spinal cord by self-assembling peptide nanofiber scaffold," Nanomedicine: Nanotechnology, Biology and Medicine, vol. 3, no. 4, pp. 311-321, 2007.

[62] V. M. Tysseling-Mattiace et al., "Self-assembling nanofibers inhibit glial scar formation and promote axon elongation after spinal cord injury," Journal of Neuroscience, vol. 28, no. 14, pp. 3814-3823, 2008.

[63] B. Chen et al., "Repair of spinal cord injury by implantation of bfgfincorporated hema-moetacl hydrogel in rats," Scientific Reports (Nature Publisher Group), vol. 5, p. 9017, 2015.

[64] U. Milbreta et al., "Three-dimensional nanofiber hybrid scaffold directs and enhances axonal regeneration after spinal cord injury," ACS Biomaterials Science \& Engineering, vol. 2, no. 8, pp. 1319-1329, 2016.

[65] L. H. Nguyen et al., "Three-dimensional aligned nanofibers-hydrogel scaffold for controlled non-viral drug/gene delivery to direct axon regeneration in spinal cord injury treatment," Scientific Reports, vol. 7 , p. 42212, 2017.

[66] V. Gautam et al., "Engineering highly interconnected neuronal networks on nanowire scaffolds," Nano letters, vol. 17, no. 6, pp. 33693375, 2017.

[67] P. Timashev et al., " $3 \mathrm{~d}$ in vitro platform produced by two-photon polymerization for the analysis of neural network formation and function," Biomedical Physics \& Engineering Express, vol. 2, no. 3, p. 035001, 2016 
[68] T. B. DeMarse et al., "Feed-forward propagation of temporal and rate information between cortical populations during coherent activation in engineered in vitro networks," Frontiers in neural circuits, vol. 10 , p. 32, 2016.

[69] A. Gladkov et al., "Design of cultured neuron networks in vitro with predefined connectivity using asymmetric microfluidic channels," Scientific Reports, vol. 7, no. 1, p. 15625, 2017.

[70] P. Assinck et al., "Cell transplantation therapy for spinal cord injury," Nature Neuroscience, vol. 20, no. 05, pp. 637-647, 2017.

[71] I. Vismara et al., "Current options for cell therapy in spinal cord injury," Trends in Molecular Medicine, vol. 23, no. 9, pp. 831-849, 2017.

[72] F.-M. Chen and X. Liu, "Advancing biomaterials of human origin for tissue engineering," Progress in polymer science, vol. 53, pp. 86-168, 2016.

[73] S. Rajangam et al., "Wireless cortical brain-machine interface for whole-body navigation in primates," Scientific reports, vol. 6, p. 22170, 2016.

[74] T. Boraud et al., "From single extracellular unit recording in experimental and human parkinsonism to the development of a functional concept of the role played by the basal ganglia in motor control," Progress in neurobiology, vol. 66, no. 4, pp. 265-283, 2002.

[75] J. R. Wolpaw et al., "Brain-computer interfaces for communication and control," Clinical neurophysiology, vol. 113, no. 6, pp. 767-791, 2002.

[76] K. Ganguly, "An introduction to brain-machine interfaces."

[77] F. Cincotti et al., "High-resolution eeg techniques for brain-computer interface applications," Journal of neuroscience methods, vol. 167, no. 1, pp. 31-42, 2008.

[78] D. A. Craig and H. Nguyen, "Adaptive eeg thought pattern classifier for advanced wheelchair control," in 2007 29th Annual International Conference of the IEEE Engineering in Medicine and Biology Society. IEEE, 2007, pp. 2544-2547.

[79] E. C. Leuthardt et al., "Electrocorticography-based brain computer interface-the seattle experience," IEEE Transactions on Neural Systems and Rehabilitation Engineering, vol. 14, no. 2, pp. 194-198, 2006.

[80] E. C. Leuthardt et al., "A brain-computer interface using electrocorticographic signals in humans," Journal of neural engineering, vol. 1, no. 2 , p. 63,2004

[81] G. Schalk et al., "Decoding two-dimensional movement trajectories using electrocorticographic signals in humans," Journal of neural engineering, vol. 4, no. 3, p. 264, 2007.

[82] M. Spüler et al., "Decoding of motor intentions from epidural ecog recordings in severely paralyzed chronic stroke patients," Journal of neural engineering, vol. 11, no. 6, p. 066008, 2014.

[83] W. Wang et al., "An electrocorticographic brain interface in an individual with tetraplegia," PloS one, vol. 8, no. 2, p. e55344, 2013

[84] G. Hotson et al., "Individual finger control of a modular prosthetic limb using high-density electrocorticography in a human subject," Journal of neural engineering, vol. 13, no. 2, p. 026017, 2016.

[85] J. M. Carmena et al., "Learning to control a brain-machine interface for reaching and grasping by primates," PLoS biol, vol. 1, no. 2, p. e42, 2003.

[86] R. D. Flint et al., "Long term, stable brain machine interface performance using local field potentials and multiunit spikes," Journal of neural engineering, vol. 10, no. 5, p. 056005, 2013.

[87] K. So et al., "Subject-specific modulation of local field potential spectral power during brain-machine interface control in primates," Journal of neural engineering, vol. 11, no. 2, p. 026002, 2014.

[88] N. J. Hill et al., "Recording human electrocorticographic (ecog) signals for neuroscientific research and real-time functional cortical mapping," JoVE (Journal of Visualized Experiments), no. 64, pp. e3993-e3993, 2012.

[89] G. Schalk and E. C. Leuthardt, "Brain-computer interfaces using electrocorticographic signals," IEEE reviews in biomedical engineering, vol. 4, pp. 140-154, 2011

[90] W. Wang et al., "Human motor cortical activity recorded with microecog electrodes, during individual finger movements," in Engineering in Medicine and Biology Society, 2009. EMBC 2009. Annual International Conference of the IEEE. IEEE, 2009, pp. 586-589.

[91] Z. C. Chao et al., "Long-term asynchronous decoding of arm motion using electrocorticographic signals in monkey," Frontiers in neuroengineering, vol. 3, p. 3, 2010.

[92] K. C. Cheung, "Implantable microscale neural interfaces," Biomedical microdevices, vol. 9, no. 6, pp. 923-938, 2007.

[93] B. Gunasekera et al., "Intracortical recording interfaces: current challenges to chronic recording function," ACS chemical neuroscience, vol. 6 , no. 1 , pp. $68-83,2015$.
[94] D. J. Krusienski et al., "An evaluation of autoregressive spectral estimation model order for brain-computer interface applications," in 2006 International Conference of the IEEE Engineering in Medicine and Biology Society. IEEE, 2006, pp. 1323-1326.

[95] T. J. Cleophas et al., Machine learning in medicine. Springer, 2013.

[96] E. Alpaydin, "Introduction to machine learning (adaptive computation and machine learning series)," The MIT Press Cambridge, 2004.

[97] C. M. Biship, "Pattern recognition and machine learning (information science and statistics)," 2007.

[98] C. Brunner et al., "Improved signal processing approaches in an offline simulation of a hybrid brain-computer interface," Journal of neuroscience methods, vol. 188, no. 1, pp. 165-173, 2010.

[99] S. Marsland, Machine learning: an algorithmic perspective. Chapman and Hall/CRC, 2011.

[100] L. F. Nicolas-Alonso and J. Gomez-Gil, "Brain computer interfaces, a review," Sensors, vol. 12, no. 2, pp. 1211-1279, 2012.

[101] D. Barber, Bayesian reasoning and machine learning. Cambridge University Press, 2012.

[102] I. Goodfellow et al., Deep learning. MIT press, 2016.

[103] Y. LeCun et al., "Deep learning," Nature, vol. 521, no. 7553, pp. 436444, 2015.

[104] J. Dube, "Fatigue resistibility and stimulus strength using intraspinal microstimulation vs. intramuscular stimulation in a rat model: Case study," 2014

[105] P. J. Grahn et al., "Wireless control of intraspinal microstimulation in a rodent model of paralysis," Journal of neurosurgery, vol. 123, no. 1 , pp. 232-242, 2015.

[106] K. Deisseroth, "Optogenetics," Nature methods, vol. 8, no. 1, p. 26, 2011.

[107] G. Courtine et al., "Transformation of nonfunctional spinal circuits into functional states after the loss of brain input," Nature neuroscience, vol. 12, no. 10, pp. 1333-1342, 2009.

[108] N. Wenger et al., "Closed-loop neuromodulation of spinal sensorimotor circuits controls refined locomotion after complete spinal cord injury," Science translational medicine, vol. 6, no. 255, pp. 255ra133255ra133, 2014.

[109] J. A. Bamford et al., "The effects of intraspinal microstimulation on spinal cord tissue in the rat," Biomaterials, vol. 31, no. 21, pp. 5552$5563,2010$.

[110] V. Caggiano et al., "Rostro-caudal inhibition of hindlimb movements in the spinal cord of mice," PloS one, vol. 9, no. 6, p. e100865, 2014

[111] V. Caggiano et al., "An optogenetic demonstration of motor modularity in the mammalian spinal cord," Scientific reports, vol. 6, p. 35185 , 2016.

[112] K. L. Montgomery et al., "Beyond the brain: Optogenetic control in the spinal cord and peripheral nervous system," Science translational medicine, vol. 8, no. 337, pp. 337rv5-337rv5, 2016.

[113] S. I. Park et al., "Soft, stretchable, fully implantable miniaturized optoelectronic systems for wireless optogenetics," Nature biotechnology, vol. 33, no. 12, p. 1280, 2015.

[114] K. L. Montgomery et al., "Wirelessly powered, fully internal optogenetics for brain, spinal and peripheral circuits in mice," Nature methods, vol. 12, no. 10, pp. 969-974, 2015.

[115] M. J. Weber et al., "A miniaturized ultrasonically powered programmable optogenetic implant stimulator system," in Biomedical Wireless Technologies, Networks, and Sensing Systems (BioWireleSS), 2016 IEEE Topical Conference on. IEEE, 2016, pp. 12-14.

[116] A. Arbabian et al., "Sound technologies, sound bodies: Medical implants with ultrasonic links," IEEE Microwave Magazine, vol. 17, no. 12, pp. 39-54, 2016.

[117] B. Pfingst et al., "Response plasticity of neurons in auditory cortex of the rhesus monkey," Experimental Brain Research, vol. 29, no. 3-4, pp. 393-404, 1977.

[118] K. C. Larsson et al., "Organic bioelectronics for electronic-to-chemical translation in modulation of neuronal signaling and machine-to-brain interfacing," Biochimica et Biophysica Acta (BBA)-General Subjects, vol. 1830, no. 9, pp. 4334-4344, 2013.

[119] S. P. Beeby et al., "Energy harvesting vibration sources for microsystems applications," Measurement science and technology, vol. 17, no. 12, p. R175, 2006.

[120] A. N. Pisarchik et al., "From novel technology to novel applications: Comment on "an integrated brain-machine interface platform with thousands of channels" by elon musk and neuralink," Journal of medical Internet research, vol. 21, no. 10, p. e16356, 2019.

[121] C. Mead, "Neuromorphic electronic systems," Proceedings of the IEEE, vol. 78 , no. 10 , pp. $1629-1636,1990$. 
[122] S. Saïghi et al., "Plasticity in memristive devices for spiking neural networks," Frontiers in neuroscience, vol. 9, p. 51, 2015.

[123] T. Otero et al., "Biomimetic electrochemistry from conducting polymers. a review: artificial muscles, smart membranes, smart drug delivery and computer/neuron interfaces," Electrochimica Acta, vol. 84, pp. 112-128, 2012.

[124] D. T. Simon et al., "An organic electronic biomimetic neuron enables auto-regulated neuromodulation," Biosensors and Bioelectronics, vol. 71, pp. 359-364, 2015.

[125] C. Ghez et al., "Roles of proprioceptive input in the programming of arm trajectories," in Cold spring harbor symposia on quantitative biology, vol. 55. Cold Spring Harbor Laboratory Press, 1990, pp. 837-847.

[126] N. G. Hatsopoulos and J. P. Donoghue, "The science of neural interface systems," Annual review of neuroscience, vol. 32, pp. 249-266, 2009.

[127] A. Ramos-Murguialday et al., "Proprioceptive feedback and brain computer interface (bci) based neuroprostheses," PloS one, vol. 7, no. 10, p. e47048, 2012.

[128] G. S. Dhillon and K. W. Horch, "Direct neural sensory feedback and control of a prosthetic arm," IEEE transactions on neural systems and rehabilitation engineering, vol. 13, no. 4, pp. 468-472, 2005.

[129] K. Horch et al., "Object discrimination with an artificial hand using electrical stimulation of peripheral tactile and proprioceptive pathways with intrafascicular electrodes," IEEE Transactions on Neural Systems and Rehabilitation Engineering, vol. 19, no. 5, pp. 483-489, 2011.

[130] D. J. Tyler and D. M. Durand, "Functionally selective peripheral nerve stimulation with a flat interface nerve electrode," IEEE Transactions on Neural Systems and Rehabilitation Engineering, vol. 10, no. 4, pp. 294-303, 2002.

[131] R. C. Rollings et al., "Ion selectivity of graphene nanopores," Nature communications, vol. 7, 2016

[132] D. C. Elias et al., "Control of graphene's properties by reversible hydrogenation: evidence for graphane," Science, vol. 323, no. 5914, pp. 610-613, 2009.

[133] R. R. Nair et al., "Fluorographene: A two-dimensional counterpart of teflon," small, vol. 6, no. 24, pp. 2877-2884, 2010.

[134] N. Li et al., "The promotion of neurite sprouting and outgrowth of mouse hippocampal cells in culture by graphene substrates," Biomaterials, vol. 32, no. 35, pp. 9374-9382, 2011.

[135] A. Fabbro et al., "Graphene-based interfaces do not alter target nerve cells," ACS nano, vol. 10, no. 1, pp. 615-623, 2016.

[136] D. Kuzum et al., "Transparent and flexible low noise graphene electrodes for simultaneous electrophysiology and neuroimaging," Nature communications, vol. 5, no. 1, pp. 1-10, 2014.

[137] B. Blaschke et al., "Mapping brain activity with flexible graphene micro-transistors. 2d mater," 2017.

[138] Y. Lu et al., "Graphene-based neurotechnologies for advanced neural interfaces," Current Opinion in Biomedical Engineering, vol. 6, pp. 138-147, 2018.

[139] Y. Lu et al., "Flexible neural electrode array based-on porous graphene for cortical microstimulation and sensing," Scientific reports, vol. 6, p. 33526, 2016.

[140] L. H. Hess et al., "Graphene transistors for bioelectronics," Proceedings of the IEEE, vol. 101, no. 7, pp. 1780-1792, 2013.

[141] Y. Huang et al., "Nanoelectronic biosensors based on cvd grown graphene," Nanoscale, vol. 2, no. 8, pp. 1485-1488, 2010.

[142] J. Zhang et al., "Communication channel analysis and real time compressed sensing for high density neural recording devices," IEEE Transactions on Circuits and Systems, vol. 63, no. 5, pp. 599-608, 2016.

[143] S. Kim et al., "Thermal impact of an active 3-d microelectrode array implanted in the brain," IEEE Transactions on Neural Systems and Rehabilitation Engineering, vol. 15, no. 4, pp. 493-501, 2007.

[144] K. Abdelhalim et al., "64-channel uwb wireless neural vector analyzer soc with a closed-loop phase synchrony-triggered neurostimulator," IEEE Journal of Solid-State Circuits, vol. 48, no. 10, pp. 2494-2510, Oct 2013.

[145] O. Novak et al., "Wireless ultra-wide-band data link for biomedical implants," in 2009 Ph.D. Research in Microelectronics and Electronics, July 2009, pp. 352-355.

[146] K. M. S. Thotahewa et al., "Power efficient ultra wide band based wireless body area networks with narrowband feedback path," IEEE Transactions on Mobile Computing, vol. 13, no. 8, pp. 1829-1842, Aug 2014.

[147] J. Zhang et al., "Communication channel analysis and real time compressed sensing for high density neural recording devices," IEEE
Transactions on Circuits and Systems I: Regular Papers, vol. 63, no. 5, pp. 599-608, May 2016.

[148] S. Kim et al., "Preliminary study of the thermal impact of a microelectrode array implanted in the brain," in Proceedings of the 28th IEEE EMBS Annual International Conference. IEEE, Aug. 2006.

[149] K. Ture et al., "Area and power efficient ultra-wideband transmitter based on active inductor," IEEE Transactions on Circuits and Systems II: Express Briefs, vol. 65, no. 10, pp. 1325-1329, 2018.

[150] K. K. Tokgoz et al., "A 120gb/s 16qam cmos millimeter-wave wireless transceiver," in 2018 IEEE International Solid-State Circuits Conference-(ISSCC). IEEE, 2018, pp. 168-170.

[151] H. Ando et al., "Wireless multichannel neural recording with a 128mbps uwb transmitter for an implantable brain-machine interfaces," IEEE Transactions on Biomedical Circuits and Systems, vol. 10, no. 6 , pp. 1068-1078, Dec 2016.

[152] S. Kabiri Ameri et al., "Graphene electronic tattoo sensors," ACS nano, vol. 11, no. 8, pp. 7634-7641, 2017.

[153] S. Lim et al., "Transparent and stretchable interactive human machine interface based on patterned graphene heterostructures," Advanced Functional Materials, vol. 25, no. 3, pp. 375-383, 2015.

[154] M. Kang et al., "Graphene-based three-dimensional capacitive touch sensor for wearable electronics," ACS nano, vol. 11, no. 8, pp. 79507957, 2017.

[155] Q. Zhang et al., "Human-like sensing and reflexes of graphene-based films," Advanced Science, vol. 3, no. 12, 2016.

[156] H.-L. C. Kao et al., "Duoskin: rapidly prototyping on-skin user interfaces using skin-friendly materials," in Proceedings of the 2016 ACM International Symposium on Wearable Computers. ACM, 2016, pp. $16-23$.

[157] X. Huang et al., "Binder-free highly conductive graphene laminate for low cost printed radio frequency applications," Applied Physics Letters, vol. 106, no. 20, p. 203105, 2015.

[158] D. Seo et al., "Wireless recording in the peripheral nervous system with ultrasonic neural dust," Neuron, vol. 91, no. 3, pp. 529-539, 2016.

[159] G. E. Santagati and T. Melodia, "Experimental evaluation of impulsive ultrasonic intra-body communications for implantable biomedical devices," IEEE Transactions on Mobile Computing, vol. 16, no. 2, pp. 367-380, 2017.

[160] S. C. O'Hern et al., "Selective ionic transport through tunable subnanometer pores in single-layer graphene membranes," Nano letters, vol. 14, no. 3, pp. 1234-1241, 2014.

[161] W. Gerstner and W. M. Kistler, Spiking neuron models: Single neurons, populations, plasticity. Cambridge university press, 2002.

[162] X. Li et al., "Remarkable conductive anisotropy of metallic microcoil/pdms composites made by electric field induced alignment," ACS applied materials \& interfaces, vol. 9, no. 2, pp. 1593-1601, 2017.

[163] F. Xu et al., "Riemann surfaces of carbon as graphene nanosolenoids," Nano letters, vol. 16, no. 1, pp. 34-39, 2015.

[164] Y. Tian et al., "Design and dynamics of a 3-dof flexure-based parallel mechanism for micro/nano manipulation," Microelectronic engineering, vol. 87, no. 2, pp. 230-241, 2010.

[165] S. Kralj and D. Makovec, "Magnetic assembly of superparamagnetic iron oxide nanoparticle clusters into nanochains and nanobundles," ACS nano, vol. 9, no. 10, pp. 9700-9707, 2015.

[166] T. Khan et al., "Nanosensor networks for smart health care," in Nanosensors for Smart Cities. Elsevier, 2020, pp. 387-403.

[167] F. Akhtar and M. H. Rehmani, "Energy harvesting for self-sustainable wireless body area networks," IT Professional, vol. 19, no. 2, pp. 32 40, 2017.

[168] K.-W. Yang et al., "Challenges in scaling down of free-floating implantable neural interfaces to millimeter scale," IEEE Access, vol. 8 , pp. $133295-133320,2020$.

[169] O. B. Akan et al., "Internet of hybrid energy harvesting things," IEEE Internet of Things Journal, vol. 5, no. 2, pp. 736-746, 2017.

[170] M. Wagih et al., "Real-world performance of sub-1 ghz and $2.4 \mathrm{ghz}$ textile antennas for rf-powered body area networks," IEEE Access, vol. 8, pp. 133 746-133756, 2020.

[171] M. Kuscu and O. B. Akan, "Maximum likelihood detection with ligand receptors for diffusion-based molecular communications in internet of bio-nano things," IEEE transactions on nanobioscience, vol. 17, no. 1 , pp. 44-54, 2018

[172] I. Akyildiz et al., "The internet of bio-nano things," IEEE Com. Mag., vol. 53, no. 3, pp. 32-40, 2015

[173] S. Furber, "Large-scale neuromorphic computing systems," Journal of neural engineering, vol. 13, no. 5, p. 051001, 2016. 
[174] M. Cognolato et al., "Gaze, visual, myoelectric, and inertial data of grasps for intelligent prosthetics," Scientific Data, vol. 7, no. 1, pp. $1-15,2020$.

[175] T. Garry and T. Harwood, "Cyborgs as frontline service employees: a research agenda," Journal of Service Theory and Practice, 2019. 